\title{
L'INFLUENCE DE LA PENSÉE DE LANGUE FRANÇAISE DANS LE CHAMP DES SCIENCES ADMINISTRATIVES BRÉSILIENNES: UN REGARD FRANCO-QUÉBÉCOIS
}

\author{
Influência do pensamento de língua francesa na academia brasileira de Administração: Um \\ olhar Franco-quebequense
}

The influence of French language thought on the Brazilian Administrative Sciences: A Quebec French look

\author{
Jean-François Chanlat',2,3 | jean-francois.chanlat@dauphine.psl.eu | ORCID : 00oo-0003-1878-4707 \\ ${ }^{1}$ Université Paris-Dauphine PSL, Dauphine Recherches en Management, Paris, France \\ 2 Institut Mines-Télécom Business School, Paris, France \\ ${ }^{3}$ HEC-Montréal, Départment of Management, Montréal, Canada
}

Texte soumis pour le Soixantième anniversaire de la RAE-Revista da Administraço de Empresas, FGV EAESP; il est dédié à tous les collègues brésiliens et de langue française qui ont contribué à ces échanges au cours de ces dernières décennies.

\section{RESUME}

L'objet de cet article est de jeter un regard sur l'influence que les travaux de langue française ont exercé sur la communauté académique brésilienne au cours des dernières décennies à partir de la connaissance que l'auteur en a, suite à une longue fréquentation du champ des sciences administratives brésilien. Pour ce faire, l'article se divise en trois parties. La première procède à un rappel de la relation que l'auteur a établie avec la communauté brésilienne pour situer qui parle et d'où il parle. La deuxième aborde brièvement le lien historique que le Brésil entretient avec la pensée française en général. Et la troisième présente les principaux courants qui semblent, selon l'auteur, avoir retenu l'attention des chercheurs brésiliens en sciences administratives. Il conclut cette réflexion par quelques considérations sur l'avenir du champ et quelques souhaits sur la relation future entre chercheurs brésiliens et chercheurs de langue française.

MOTS CLÉS | Sciences administratives, Brésil, langue française, France, Québec.

\section{RESUMO}

O objetivo deste artigo é dar uma olhada na influência que as obras de língua francesa têm exercido sobre a comunidade acadêmica brasileira nas últimas décadas, a partir do conhecimento do autor e sua longa associação do campo das ciências administrativas brasileiras. Para isso, o artigo está dividido em três partes. A primeira lembra a relação que o autor estabeleceu com a comunidade brasileira para localizar quem está falando e onde ele está falando. A segunda discute brevemente o vínculo histórico que o Brasil mantém, de um modo geral, com o pensamento francês. A terceira apresentará as principais correntes intelectuais que parecem, segundo o autor, ter chamado a atenção dos pesquisadores brasileiros em ciências administrativas. Esta reflexão se conclui com algumas considerações sobre o futuro da área e alguns desejos sobre as futuras relações entre pesquisadores brasileiros e pesquisadores francófonos.

PALAVRAS-CHAVE / Ciências administrativas, Brasil, língua francesa, França, Québec.

\section{ABSTRACT}

The object of this article is to take a look at the influence that French-language works have had on the Brazilian academic community over the past decades, based on the author's knowledge of it, and a long attendance of the field of Brazilian administrative sciences. To do this, the article is divided into three parts. The first recalls the relationship that the author has established with the Brazilian community to locate who is speaking and from where he is speaking. The second briefly discusses the historical link that Brazil maintains with French thought in general. And the third presents the French main intellectual currents, which, according to the author, seem to have caught the attention of Brazilian researchers in administrative sciences. This reflection ends with some considerations on the future of the field and some wishes on the future relationship between Brazilian researchers and French-speaking researchers.

KEYWORDS I Administrative science, Brazil, French language, France, Quebec. 
Ce numéro spécial du soixantième anniversaire de la $R A E$, auquel m'a cordialement invité à contribuer sa rédactrice-en-chef, Maria José Tonelli, est pour moi non seulement un honneur et un témoignage de la longue relation que j'entretiens avec la communauté brésilienne des sciences administratives, depuis plus de trente ans, mais aussi plus généralement, une reconnaissance des liens intellectuels et amicaux qui se sont tissés au cours de toutes ces années avec la communauté des chercheurs francophones à laquelle j'appartiens. C'est donc au nom de cette appartenance intellectuelle et linguistique que je vais porter à la demande des organisateurs de ce numéro, un regard sur l'influence que les travaux de langue française ont exercé sur la communauté académique brésilienne au cours des dernières décennies. Je le fais dans un contexte historique très particulier: celui d'une pandémie mondiale qui interpelle notre mode de développement, et se révèle une véritable crise anthropologique (Chanlat, 2020).

Mon article se divisera en trois parties. La première procédera à un rappel de ma relation avec la communauté brésilienne pour rappeler qui parle et d'où je parle. La deuxième abordera brièvement le lien historique que le Brésil entretient avec la pensée française en général. Et la troisième présentera les principaux courants qui semblent, selon moi, avoir retenu l'attention des chercheurs brésiliens en sciences administratives. Nous conclurons cette réflexion par quelques considérations sur l'avenir du champ et quelques souhaits sur la relation future entre chercheurs brésiliens et chercheurs de langue française.

\section{MA DÉCOUVERTE DU BRÉSIL: UN BREF RAPPEL BIOGRAPHIQUE}

Avant de connaître le pays dans sa réalité sensible, j'avais à l'instar de beaucoup d'autres personnes dans le monde, une vision du Brésil qui relevait en partie de l'image souvent stéréotypée qu'on peut en avoir: l'Amazonie, le Carnaval de Rio, la plage de Copacabana, la Samba, le football, enfant j'avais en effet assisté à la retransmission télévisée de la demi-finale de la Coupe du monde de 1958, une première pour l'époque, entre la France et le Brésil, et à l'émergence de Pelé comme un des grands joueurs de l'histoire de ce sport, et avais bien sûr vu en 1964 le célèbre film français, l'Homme de Rio, avec Jean-Paul Belmondo. Mais, j'en avais aussi acquise une autre qui venait de certaines connaissance de son histoire et de ses particularités sociologiques recueillies, lors de mes longues années vécues à HEC-Montréal, auprès d'un collègue et ami, Allain Joly (2004), qui faisait ses études doctorales à la Fundação Getulio Vargas, Escola de Administração de Empresas de São Paulo (FGV EAESP), au début des années 80, et qui allait jouer un rôle déterminant dans ma relation avec le Brésil.

Ma première rencontre avec un enseignant brésilien dans le champ des sciences administratives, je la fis à Montréal au cours de l'année universitaire 1984-1985. Alors, jeune professeur à HEC-Montréal, je faisais connaissance de Roberto Fachin, Professeur à la faculté d'administration de l' Universidade Federal do Rio Grande do Sul (UFRGS). Déjà, une figure dans le champ brésilien, Roberto était venu passer une année sabbatique. Tout au long de cette année universitaire 84-85, nous avons donc longuement échangé à la fois sur la situation brésilienne en général, et sur l'enseignement de la gestion en particulier, notamment lors d'un colloque international important organisé au printemps 1985 par mon frère Alain et Maurice Dufour sur cette thématique.

En septembre 1990, je rencontrais de nouveau à Montréal, grâce à la médiation de Allain Joly, deux professeurs importants de la FGV EAESP, les Professeurs Ofélia Torres et Fernando Prestes Motta, lesquels étaient venus assister au grand colloque international que j'avais organisé autour de l'ouvrage que je venais de coor- 
donner (Chanlat, 1990) suite à mon année sabbatique en France, et qui allait devenir rapidement un ouvrage de référence dans les pays de langue française (Nugent, 1993; Padioleau, 1992; Sciences Humaines, 1998).

Suite à leur participation et à l'intérêt que les professeurs Torres et Motta allaient avoir pour la perspective développée dans cet ouvrage considéré aujourd'hui comme pionnier (Bouville \& Yousfi, 2021), et au soutien financier apporté par le Centre d'étude en administration internationale (CETAI) des HEC-Montréal pour la traduction, une édition portugaise en trois tomes allait être rapidement organisée au Brésil par Ofélia Torres, ses différents chapitres étant traduits par un ensemble de professeurs brésiliens, notamment de la FGV EAESP (Chanlat, 1992, 1994, 1996). Pour la sortie du premier tome en 1992, je fus donc invité, à l'instigation de Ofélia Torres, de Roberto Fachin et de Taniâ Fischer avec l'appui du CNPq à faire, cette année-là, une tournée de conférences au Brésil.

Ce premier séjour brésilien de trois semaines me conduira successivement à la FGV EAESP, aux facultés d'administration des universités fédérales du Minais Gerais, de Rio Grande do Sul et de Bahia et à la Pontifícia Universidade Católica do Rio de Janeiro (PUC-Rio); elle va me permettre de découvrir le Brésil, ce pays-continent, dans toute sa diversité humaine et géographique et ses fractures sociales. C'est à l'occasion de ces voyages, qui se multiplieront par la suite, que je vais rencontrer de nombreux collègues: Valmiria Carolina Piccinini, Ana Maria Kirschner, Maria Elisabeth Antunes Lima, Suzana Braga Rodrigues, Liliana Segnini, Marlene Catarina de Oliveira Lopes Melo, Alfredo Alves de Oliveira Melo, Juvêncio Braga de Lima, Tânia Fischer, José Antônio Gomes de Pinho, Silvia Vergara, Patricia Tomei, Clovis Machado †, Maria José Tonelli, Fernando Prestes Motta †, Sigmar Malvezzi, Maria Irene Betiol, Thomaz Wood Jr, Miguel Caldas, Bianor Calvacanti, Eduardo Marques, Yann Duzert, Maria Ester de Freitas, Maria Teresa Fleury, Roberto Lima Ruas, Luiz Bignetti †, Fábio Bittencourt Meira, Isabella de Vasconcelos, Flávio de Vasconcelos, Angelo Soares, Guilherme Azevedo, Marlei Pozzebon, Eduardo Diniz, Rafael Alcadipani, Sidinei Rocha-de-Oliveira, qui deviendront par la suite des amis; et certains de mes futurs étudiants et étudiantes: Eduardo Davel, Gelson Junquilho, Jair Nascimento Santos, Carolina Andion, le regretté José Roberto Gomes da Silva †, Maria Elisa Brandao, Marina Nakayama, Marcelo Dantas, Mauricio Serva, avec lesquels j'entretiens depuis des relations intellectuelles et amicales.

Au cours de toutes ces années, passées à Montréal, à Strabourg, puis à Paris, à partir de mon installation définitive à Dauphine en septembre 2001, je vais revenir régulièrement au Brésil comme conférencier invité à des congrès de l'Encontro da ANPAD (EnANPAD), d'Encontro de Estudos Organizacionais da ANPAD (EnEO), de l'IberoAcademy of Management, aux colloques internationaux de Universidade Federal da Bahia (UFBA) sur le pouvoir local, organisés à Salvador par la Professeure Tânia Fischer et le Núcleo de Estudos sobre Poder e Organizações Locais (NEPOL), aujourd'hui le Centro Interdisciplinar de Desenvolvimento e Gestão Social (CIAGS), à un colloque de la Pontifícia Universidade Católica do Rio Grande do Sul (PUC-RS) de Porto Alegre, et à donner des séminaires et des conférences dans de nombreuses institutions publiques et privées à São Paulo, Belo Horizonte, Campinas, Brasília, São Leopoldo, Florianópolis, Porto Alegre, Salvador, Rio de Janeiro, Vitória... Je vais d'ailleurs en profiter à quelques reprises pour participer à plusieurs jurys de thèse, voire co-superviser certains travaux doctoraux, dont celui du regretté José Roberto Gomes da Silva † (2010), dont la disparition tragique a été très associée, comme beaucoup le savent, à cette relation franco-brésilienne (Chanlat, 2010b). Cette activité d'encadrement de chercheurs brésiliens va se poursuivre ensuite à Paris où j'ai encadré dans un Master recherche, le mémoire de Simone Cota, dirigé la thèse de doctorat de Virginia Drummond, superviser lors de son séjour post-doctoral, Rodrigo Bandeira de Mello, qui allait être associé annuellement par la suite à notre unité d'enseignement et de recherche, et d'où j'ai pu participer aux jurys de thèse de doctorat de Teresa Bicalho de Menezes, une autre doc- 
torante brésilienne de Dauphine, dirigée par mon collègue, le professeur Jacques Richard, et à ceux de Bibiana Volker Martins et de Laura Scherer, doctorantes à I'UFRGS de Porto Alegre, que j'avais reçues préalablement à Paris dans le cadre d'une bourse sandwich.

Au cours de toutes ces années, plusieurs autres de mes ouvrages seront traduits (Chanlat, 2000, 2010a), certains de mes articles, publiés dans les revues: RAE, Organização e Sociedade, Gestão e Planejamento, Cadernos EBAPE.BR, GV Executivo, et des chapitres, paraîtront dans plusieurs autres ouvrages (Bendassolli \& Soboll, 2010/2021; Davel \& Melo, 2005; Davel \& Vergara, 2001). Enfin, je vais être amené à co-coordonner avec mes collègues et amis, Roberto Fachin et Tânia Fischer, deux importants ouvrages: Análise das organizações: perspectivas latinas. Vol 1 e 2, qui reprendront un certain nombre de communications faites sur le même thème au cours d'un colloque historique, tenu en juillet 2000 à Zacatecas au Mexique, organisé par nos collègues et amis mexicains des études organisationnelles de la UAM-Iztapalapa ((Ramirez Martinez \& Gonzáles-Miranda, 2018; Gonzáles-Miranda \& Ramirez Martinez, 2018, 2020) et moi-même, et qui avait réuni pour l'occasion des collègues reconnus de divers pays francophones (France, Québec, Belgique), hispanophones (Mexique, Colombie, Argentine) et du Brésil (Chanlat, Fachin, \& Fischer, 2006, 2007; Fachin, 2014).

Comme on peut le voir, c'est ces nombreux séjours en terre brésilienne qui m'ont permis peu à peu de me familiariser avec le pays et d'acquérir une bonne vision du champ des sciences administratives, une vision franco-québécoise, compte tenu de ma propre trajectoire intellectuelle et professionnelle. A chaque fois, ils m'ont permis de constater combien la pensée française y était prise au sérieux, et, combien les travaux de langue française dans le domaine des organisations, du travail et de l'économie suscitaient de l'intérêt.

\section{L'OUVERTURE BRÉSILIENNE À LA PENSÉE FRANÇAISE: UNE HISTOIRE ANCIENNE}

Quand je suis arrivé, pour la première fois, au Brésil, j'ai en effet constaté très vite l'influence que la pensée française avait eue historiquement, et avait encore sur la société brésilienne. J'ai été notamment frappé par le nombre de collègues en sciences administratives qui maitrisaient la langue française ou la comprenaient sans toujours la parler.

Selon certains analystes brésiliens et étrangers, cette influence française remonte au XVIIle siècle avec celle que va exercer à cette époque la pensée des philosophes français des Lumières, ensuite, elle va se poursuivre au XIXe siècle avec la diffusion de la pensée de Auguste Comte sous l'impulsion de Miguel de Lemos, de Benjamin Constant Botelho de Magalhaes et de Raimundo Teixeira Mendes (Grange, 2000; Lins, 1967), dont j'ai pu voir encore des vestiges architecturaux de la première église positiviste à Rio, érigé en 1881, quelques années avant la proclamation de la République brésilienne; et dont on peut en retrouver une trace éloquente dans la devise officielle du Brésil "Ordre et Progrès" qui reprend la devise comtienne (Tyr, 2007). La philosophie de Comte étant alors vue comme une source d'inspiration politique pour les élites modernistes qui aspiraient à fonder une République et à mettre fin à l'esclavage, comme le souligne Lorelai Kury à ce sujet:

Le projet politique positiviste, républicain et autoritaire, a été fort influent au Brésil, particulièrement puissant dans le tournant du XIXème siècle au XXème siècle. Les disciples d'Auguste Comte ont même vaincu, en 1889, la bataille symbolique autour du drapeau national républicain: le modèle proposé par Raimundo Teixeira Mendes, ajoutait à l'ancien 
drapeau de l’Empire la devise « Ordem e Progresso » (Ordre et Progrès), censée lier le passé à l'avenir, ce qui suivait de près les instructions du philosophe français. Même si le positivisme a perdu au long du XXème siècle l'influence anciennement active, le courant a pourtant laissé des traces idéologiques profondes dans les classes moyennes urbaines du pays. (Kury, 2003, p. 126)

Au XXe siècle, cette influence française continuera à perdurer. Elle sera liée à la création dans les années trente de l' Universidade de São Paulo (USP) à laquelle seront associées des personnalités françaises marquantes comme les géographes Pierre Monbeig et Pierre Deffontaines, l'historien Fernand Braudel, l'ethnologue Claude Lévi-Strauss, le sociologue et anthropologue Roger Bastide, le philosophe François Châtelet, les historiens Gérard Lebrun et Jean-Pierre Vernant, l'épistémologue Gilles-Gaston Granger, et plus généralement, à la forte influence qu'exercera la philosophie française, discipline phare au Brésil, tout au long de ce siècle.

Comme le rappelait Sergio Paulo Rouanet dans le cadre de l'année du Brésil organisée en France, en 2010:

L'influence française a été tellement prédominante qu'une histoire de la réception des idées philosophiques françaises se confond dans une large mesure avec l'histoire de la philosophie au Brésil. Pour démontrer cette hégémonie, il suffit de rappeler un livre récent sur l'enseignement de la philosophie à l'Université de São Paulo (USP), l'une des plus respectées au Brésil et fondée sous l'inspiration de professeurs français. L'auteur appelle le Département de Philosophie de cette Université “Département français d'outre-mer”: c'est tout dire (2010, p. 41).

C'est ainsi que la pensée de Bergson, l'humanisme intégral de Maritain, le personnalisme de Mounier, le structuralisme de Lévi-Strauss, l'existentialisme de Sartre, et plus tard, les travaux de Castoriadis, de Foucault, de Derrida et de Deleuze vont se succéder au ryhme des tendances propres à chacune des époques concernées, et connaître une grande popularité au sein des élites intellectuelles brésiliennes. A partir des années 70, elle se conjuguera avec l'engouement que la communauté brésilienne va alors accorder aux sciences sociales françaises qui connaissent un grand rayonnement international et aux liens que certains grands intellectuels brésiliens comme Fernando Henrique Cardoso ont tissé avec de grandes figures intellectuelles françaises, notamment avec Alain Touraine (Kirschner \& Gomes, 2014b). L'arrière-plan intellectuel brésilien reste donc très marqué par ces traces historiques successives, laissées par la pensée française, même si d'autres pensées, notamment de langue anglaise et de langue allemande, vont prendre une place croissante au Brésil au cours des dernières décennies (Rouanet, 2010).

Due à l'attractivité historique qu'a donc eue la culture française au sein des élites (Fachin \& Cavedon, 2003), un phénomène que l'on peut d'ailleurs observer dans d'autres pays d'Amérique latine (Bonnafous, 1953), ce terreau historique va se révéler favorable à partir de la fin du XXe siècle, à une ouverture sur les travaux de langue française, portant sur le travail, les organisations et l'économie. Ce qui sera favorisé par le fait que de nombreux professeurs brésiliens vont venir faire leurs études doctorales en France, et ou par la suite, au Québec, à un moment où la communauté brésilienne des sciences administratives cherche par ailleurs des alternatives à la pensée anglo-saxonne dominante dans son champ (Bertero, Caldas, \& Wood, 1999; Cassundé, Barbosa \& Mendonça, 2016; Fachin, 2014; Fachin \& Cavedon, 2003; Fischer, 1985; Motta, 1990; Paula, Maranhão, Barreto, \& Klechen, 2010; Torres \& Gonçalves, 1991; Vergara, 2006; Waiandt, 2018). 


\section{LES CHERCHEURS EN SCIENCES ADMINISTRATIVES BRÉSILIENS EN QUÊTE D'ALTERNATIVE À L'HÉGÉMONIE ANGLO-SAXONNE}

Lorsqu'on consulte les travaux brésiliens, notamment ceux qui essaient de faire des synthèses du champ dans le domaine des sciences sociales (Bendassolli, \& Soboll, 2010/2021; Kirschner \& Gomes, 2014a, 2014b), et des sciences administratives (Bertero, 2006; Bertero, Alcadipani, Cabral, Faria, \& Rossoni, 2013; Bertero, Caldas, \& Wood, 2005), on observe que les travaux de langue française, malgré la domination des travaux anglo-saxons (Caldas, Fachin \& Fischer, 1999; Clegg, Hardy \& Nord, 1999, 2001, 2004), sont bien présents dans ces réflexions, notamment chez les chercheurs brésiliens en quête d'une alternative à cette domination des références anglo-américaines (Fachin, 2014; Vergara, 2006; Vergara \& Pinto, 2001; Waiandt, 2018).

Dans le champ des sciences administratives brésilien, cette hégémonie anglo-américaine date des années cinquante au moment où les gouvernements américain et brésilien signent des accords afin d'établir les premiers programmes d'enseignement et de créer les premières institutions supérieures en gestion, dans un contexte de guerre froide, avec l'aide de la Fondation Ford, et qui s'inspire très largement du modèle américain de formation en gestion (Alcadipani \& Bertero, 2012, 2014; Alacadipani \& Caldas, 2012; Alcadipani \& Cooke, 2013; Barros, Alcadipani \& Bertero, 2018; Serva \& Andion, 2021).

Au Brésil, cela s'inscrit par ailleurs dans un contexte plus large d'une société, historiquement, ouverte aux idées venant de l'extérieur, ce que les Brésiliens appellent: "O estrangeirismo" (Bartel-Radic, 2013; Buarque de Holanda, 1936; 1998; Caldas \& Wood, 1997; Freyre, 1963; Motta, Alcadipani, \& Bresler, 2001), et qui se caractérise d'ailleurs souvent dans les faits par une forme d'anthropophagie des pratiques étrangères (Davel, Dantas, \& Vergara, 2008).

De nos jours, la situation actuelle dans le champs se présente, selon certains collègues (Bertero et al., 2013) de la manière suivante:

... La grande majorité des études en administration au Brésil, notamment dans les domaines des études organisationnelles, de l'enseignement et de la recherche, consistent en des essais théoriques axés sur des auteurs européens .... Il semble qu'une grande partie des chercheurs brésiliens aient horreur d'aller sur le terrain et lorsqu'ils le font, ils produisent des textes, dont la méthodologie est souvent défaillante, et incohérente. Être à l'écoute du national, c'est aller au contact de la «réalité» brésilienne, de l'étudier et de la connaître à l'aide de références qui permettent à cette réalité de s'exprimer dans sa complexité et de ne pas être entravée et cachée derrière des théories et des modèles, dont la pertinence est souvent discutable; nous avons donc, jusqu'ici, très peu étudié l'administration au Brésil. L'une des raisons de notre manque d'originalité tient au fait que nous regardons très peu notre réalité, et ce qui se passe dans notre pays. Cependant, l'esprit de clocher de certains .... est compensé par l'attirance d'autres envers les travaux étrangers ... . On a en effet tendance à surévaluer l'étranger .... Valoriser l'enseignement et la recherche qui se fait dans le Nord, et ce, à nos dépens, joue un rôle fondamental dans la construction de notre infériorité en tant que chercheurs, cela implique en effet de laisser aux chercheurs du Nord, le rôle de créer les modèles, et de nous penser et de nous positionner comme de simples imitateurs (p.186). (Traduction de l'auteur) 
Dans ce constat sévère, ils soulignent en passant le rôle joué par le marché des publications:

Plus important encore, il faut garder à l'esprit qu'une grande partie du marché de l'édition universitaire, considérée comme «internationale», est contrôlée par des éditeurs et des universitaires anglo-saxons. Si nous prenons celles qui sont considérées comme les principales revues du domaine, nous pouvons observer que seuls des articles portant sur des thèmes particuliers publiés dans des universités spécifiques sont en effet publiés. Aujourd'hui, nous voyons de nombreux universitaires de différentes régions du monde à la recherche d'un «courtier» anglo-saxon pour que leurs articles puissent être publiés dans ces revues renommées. Ainsi, le gringo supérieur est privilégié et notre condition de subalternes demeure. Beaucoup de gringos croient, de ce fait, que nous sommes inférieurs (Bertero et al., 2013, p.186). (Traduction de l'auteur.)

En conséquence, pour ces mêmes auteurs:

Il existe une pensée hégémonique dans notre domaine, principalement d'origine nord-américaine, qui a tendance à ne pas tenir compte des perspectives qualitatives et des approches plus réflexives. Toutefois, être américain ne signifie pas de suivre toujours cette vision réductrice de la recherche. Dans notre domaine, il y a en effet des Américains qui prônent une vision plus pluraliste, tout comme nous avons des chercheurs latinos qui préconisent une vision plus restrictive de la recherche. Ce que nous percevons, c'est donc un choc international entre différentes visions. (Bertero et al., 2013, p193). (Traduction de l'auteur.).

C'est dans ce contexte historique que certains chercheurs brésiliens vont donc chercher à partir des années soixante à développer des pensées originales. C'est le cas de Guerreiro Ramos (1981/1989, 1983, 1984;Azevedo \& Albernaz, 2006; Serva, 1997a, 1997b; de Paula, 2007; Serva \& Andion, 2021), de Mauricio Tragtenberg ou de Fernando Prestes Motta (Segnini \& Alcadipani, 2014), lesquels vont s'appuyer, pour le premier, notamment sur l'humanisme intégral de Jacques Maritain et le personnalisme de Emmanuel Mounier (Azevedo \& Albernaz, 2006), et les deux derniers, sur d'autres traditions intellectuelles françaises, notamment anarchistes (Motta, 1981, 1984, 1990, 2003; Tragtenberg, 2005, 2010, 2012). Ces trois figures brésiliennes connaissant un rayonnement très important au Brésil (Harzing, 2016). D'autres, à l'instar de Fachin et Cavedon à la fin de leur article, qui portait justement sur l'influence de la pensée française au sein des études organisationnelles brésiliennes, n'hésitent pas à souligner l'intérêt de ces lectures:

En conséquence, il faut rechercher, dans la francophonie, des contributions universitaires qui, en étant basée sur certaines sphères de la connaissance, traduisent la pensée critique française si chère aux intellectuels brésiliens. Il ne fait aucun doute que la disponibilité de ces œuvres en portugais offre un réel apport pour les universitaires brésiliens qui n'ont pas reçu de formation doctorale en France ou au Québec (Fachin \& Cavedon, 2003, p.11). (Traduction de l'auteur)

Si le champ brésilien des sciences administratives a donc été influencé par de nombreux travaux de langue française, j'ai décidé, pour les besoins de cet article, d'en retenir ici, sans d'ailleurs les hiérarchiser, les trois dis- 
ciplines qui en ont été, selon moi, les sources inspiratrices, à savoir: les sciences des organisations, les sciences du travail, et les sciences économiques hétérodoxes. Ce phénomène ayant été encouragé par le bilinguisme de nombreux chercheurs brésiliens, et ou la traduction d'ouvrages de référence, appartenant à ces disciplines, par des éditeurs brésiliens.

\section{Les sciences des organisations}

La première source d'inspiration a été les sciences des organisations, fruit du développement des travaux associés à l'émergence des organisations comme fait social au début des années soixante (Caldas, Fachin, \& Fischer, 1999; Chanlat \& Séguin, 1987; Clegg \& Bayley, 2007; Clegg, Hardy \& Nord, 1999; 2001, 2004; Etzioni, 1967; Gonzáles-Miranda \& Ramirez Martinez, 2020; Perrow, 1976; Séguin \& Chanlat, 1983; Saussois, 2016).

Dans le contexte francophone, notamment français, cela va se traduire par un ensemble de recherches qui vont tourner autour de quatre grands axes: la psychosociologie des organisations et l'analyse institutionnelle, la sociologie des organisations et de l'entreprise, l'anthropologie des organisations et la gestion des organisations.

Parmi le grand nombre de travaux existants dans le champ francophone (Chanlat, 1992b, 1994, 2014; Stokes, Davoine \& Oiry, 2014; Entreprises et Histoire, 2016), les chercheurs brésiliens vont au sein du fort riche courant psychosociologique (Barus-Michel, Enriquez, \& Lévy, 2002), privilégier les travaux de l'analyse institutionnelle (Lapassade, 1989; Lourau, 1975; Rossi \& Passos, 2014), ceux de Eugène Enriquez (1990, 1997, 2007, 2014), de Max Pagès, Bonetti et de Gaulejac (1987), de Nicole Aubert et de Vincent de Gaulejac (1993; de Gaulejac, 2005), et de Gilles Amado (2013) comme l'ont rappelé Newton Garcia de Araújo et Andrade de Barros dans leur revue récente (2019); ces derniers vont exercer une influence sur la vision que les chercheurs brésiliens en sciences administratives vont se faire du rapport vie psychique et organisation et contribuer à inspirer, avec bien d'autres chercheurs de langue française (Didier Anzieu, Serge Moscovici, Jean-Claude Rouchy, Guy et Jacqueline Palmade, André Green, Laurent Lapierre...), le champ des études organisationnelles critiques brésiliennes à partir des années quatrevingt-dix (Bendassolli \& Soboll, 2011/2021; Freitas, 1999b; Freitas \& Motta, 2000).

Un autre courant de recherche auxquels les chercheurs brésiliens vont puiser est la sociologie des organisations. Cette discipline, fondée en France par Michel Crozier, au début des années soixante, va connaître par la suite un développement important dans les pays francophones et latins sous l'impulsion des travaux du Centre de Sociologie des Organisations (CSO), crée et dirigé par Crozier (1963a e b, 1981; 2000; Crozier \& Friedberg, 1977; Entreprises et Histoire, 2016), qui va devenir vite le foyer de formation de la plupart des pionniers francophones en la matière (Chanlat, 1992b, 1994; Saussois, 2016) et influencer certains travaux brésiliens, notamment dans le domaine de l'administration publique (de Vasconcelos \& Pinochet, 2004). Elle va également bénéficier de ceux du Laboratoire de Sociologie du changement des institutions (LSCI), crée et dirigé par Renaud Sainsaulieu (1977/2014, 1990, 1997, 2002), un des premiers collaborateurs de Crozier au CSO, et de ceux du Groupe lyonnais de sociologie industrielle (GLYSI), crée et dirigé par Philippe Bernoux (1985; Amblard, Bernoux, Herreros, \& Livian, 1995). C'est au sein de la sociologie des organisations qu'émergera, au tournant des années quatre-vingt dix, la sociologie de l'entreprise (Bélanger \& Lévesque, 1996; Dupuis \& Kuzminski, 1998; Sainsaulieu, 1990; Sainsaulieu \& Segrestin, 1987; Segrestin, 1992), laquelle va jouer un rôle important, au début des années 2000, au Brésil (Kirschner \& Gomes, 2014a; Sainsaulieu \& Kirschner, 2006).

A partir du début des années quatre-vingt-dix, un autre ensemble de travaux va connaître une grande popularité au sein des sciences administratives brésiliennes: ce sont ceux qui vont être associés à ma propre 
perspective anthropologique. On les retrouve exposés dans les trois tomes de la traduction brésilienne de l'ouvrage que j'ai dirigé: L'individu dans l'organisation: les dimensions oubliées, Les dimensions oubliées, dans deux autres ouvrages, l'un traduit par Ofelia Torres (Chanlat, 2000), et l'autre publié par Isabela de Vasconcelos dans sa collection "Debates em Administração" (Chanlat, 2010), et dans de nombreuses autres publications (Serva \& Andion, 2021). Comme le soulignaient Ofelia Torres e Marilson Alves Gonçalves (1991) dans le compterendu qu'elles faisaient de l'ouvrage en 1991:

L'ouvrage L'Individu dans l'Organisation: les dimensions oubliées présente de manière magistrale le projet d'un renouvellement des connaissances et des pratiques des sciences du comportement dans le monde des affaires. À partir d'une réflexion générale sur le développement d'une anthropologie de la condition humaine dans les organisations, chaque auteur participant à cet ouvrage développe sa propre contribution. Du langage à l'espace, en passant par l'univers symbolique, le temps, la vie psychique, l'altérité et la psychopathologie, l'œuvre en question atteint ainsi l'objectif : défendre une vision commune de l'être humain, reconnaissant l'importance de son rôle dans chaque dimension sans toutefois freiner le choc des idées et des styles et la liberté d'avoir quelque désaccord sur les méthodes et sur l'approche du thème commun. Comme il s'agit d'une publication qui ouvre de nouvelles perspectives, elle devrait intéresser autant les professeurs que les chercheurs et les étudiants en gestion, en particulier les étudiants de maîtrise et de doctorat, nombreux aujourd'hui au Brésil, car elle peut servir de référence à l'élaboration de monographies et de thèses appliquées à ce nouveau champ de connaissances. Par son contenu réflexif et pratique sur l'être humain dans les organisations, vu comme une personne vivante, concrète et comme un acteur, il ne peut manquer d'intéresser aussi les cadres d'entreprises et les responsables des ressources humaines (pp. 107-108). (Traduction de l'auteur)

Autrement dit, cette perspective va amener les chercheurs brésiliens à s'intéresser aux différentes dimensions mises de l'avant dans l'ouvrage par de nombreux collègues francophones grâce à son édition brésilienne (Fachin \& Cavedon, 2003; Torres \& Gonçalves 1992; Vergara, 2006), et à en "brésilianniser" les études (Chanlat et al., 2006); cette perspective n'étant pas ethnocentrique; elle inspirera aussi certaines réflexions brésiliennes dans le domaine de l'épistémologie des sciences administratives, en plein développement (Serva \& Andion, 2021).

Enfin, certains travaux en sciences de gestion vont aussi susciter un intérêt notable au sein de la communauté brésilienne. On peut souligner, entre autres, l'ouvrage de synthèse critique de Omar Aktouf sur le management, dans la mouvance des travaux menés à HEC-Montréal à l'époque (Chanlat \& Dufour, 1985; Chanlat, 1990), dont la traduction en portugais va connaître une grande diffusion au Brésil (1996); certains travaux du CRG de l'École Polytechnique de Paris (Berry, 1983, 2011), notamment ceux de Jacques Girin (2016) sur le langage et les situations de gestion (Bayard, Borzeix, \& Dumez, 2010), et de Hervé Dumez (2016) sur les méthodologies qualitatives; ceux de Audet et Malouin (1986), de Audet et Déry (1996), au Québec et de Martinet (1990) et de Pesqueux en France, sur l'épistémologie des sciences de gestion (Martinet \& Pesqueux, 2013; Serva, 2013, 2017 a et b) et de Pesqueux sur la philosophie et les organisations (2008); ceux de Thévenet (1992) sur la culture d'entreprise; ceux, pionniers, de Philippe d'Iribarne et de son équipe "Gestion et société" sur la culture nationale (d'Iribarne, Chevrier, Henry, Segal, \& Tréguer-Felten, 2020; d'Iribarne, Henry, Segal, Chevrier, \& Globokar, 1998); ceux de Jean-Pierre Dupuis, 
Serge Bouchard et Omar Aktouf sur la culture et la symbolique organisationnelle (Freitas, 1991; 1999a), ceux sur le leadership de Laurent Lapierre (1989, 2005), présentés notamment dans Chanlat (1992, 1994, 1996); ou encore ceux de Romain Laufer (1977) sur la crise de légitimité des organisations (Laufer \& Paradeise, 1982; Motta, 1988).

Plus récemment, on peut évoquer ceux de l'École des Mines, notamment ceux de Segrestin et Hatchuel (2012) autour de la redéfinition de l'entreprise qui vont susciter un engouement certain auprès de plusieurs chercheurs s'intéressant à la gestion des organisations solidaires (Eynaud \& França, 2019), et contribuer, en France, à redéfinir juridiquement l'entreprise (Hatchuel, 2021), un sujet qui est aussi vu comme clé par de nombreux chercheurs de langue anglaise (Clarke, O'Brien \& O'Kelley, 2019); ceux de la "Chaire Management, diversité et cohésion sociale", de l'Université Paris-Dauphine, animée par Stéphanie Dameron, Mustafa Özbilgin et moi-même pendant dix ans (2008-2018) (Chanlat et al., 2013; Chanlat \& Özbilgin, 2018, 2019), et dont les travaux et les deux Symposiums internationaux organisés à Paris en 2011 et 2015 vont avoir d'importantes répercussions brésiliennes avec la participation de Maria Ester de Frettas et de Marcelo Dantas, grâce aux contacts qu'ils auront établis à

ces occasions avec un certain nombre d'autres chercheurs. De là, émergera en effet dans le champ des sciences administratives une nouvelle sphère de recherche autour de l'interculturalité (Davel, Dupuis, \& Chanlat, 2008), et de la diversité, qui cherchera à mieux comprendre, notamment les expériences d'expatriation et réduire les inégalités et les discriminations vécues dans l'univers des organisations brésiliennes (Freitas \& Dantas, 2011; Bueno \& Freitas, 2016; Freitas, 2018; Freitas \& Dantas, 2014).

On peut enfin signaler les travaux fondamentaux menés tout récemment en comptabilité par Alexandre Rambaud et Jacques Richard qui, en interrogeant les fondements mêmes de la discipline en vue d'une refonte de notre système économique, devrait à terme intéresser les chercheurs brésiliens en quête d'un modèle de gestion écologique en raison des liens que Jacques Richard a établi avec certains chercheurs brésiliens (Rambaud \& Richard, 2021); ou encore ceux de David, Hatchuel \& Laufer travaillant à donner de nouvelles fondations aux sciences de gestion (2012). Et bien sûr, les nombreux travaux des réseaux scientifiques francophone, comme le RIODD portant sur la responsabilité des entreprises et le développement durable, l'AGRH (Gestion des ressources humaines), I'AIMS (management stratégique et organisation), RHIME (Ethique et interdisciplinarité) et ATLAS-AFMI (management international) auxquels les chercheurs brésiliens francophones peuvent avoir accès via Internet et peuvent participer; certains l'ayant parfois faits au cours des trois dernières décennies. Enfin, ils et elles peuvent enfin consulter plusieurs revues qui leur donneront accès à des travaux souvent originaux comme la Revue Française de Gestion, Gérer et Comprendre, Gestion, Management International, Management et Avenir, Finance, Contrôle, Stratégie, M@n@gement, Rhime, le Libellio d'Aegis, Entreprise et Société, Revue internationale PME, Gestion 2000, La Revue des Sciences de Gestion, Management et Sciences Sociales, Revue de l'organisation responsable, Question(s) de management, Entreprises et histoire...Et consulter le site de la Société Française de Management pour lire ses comptes rendus, ses avis et ses publications sur des questions d'actualité concernant notre champ.

\section{Les sciences du travail}

Les sciences du travail constituent la deuxième source d'inspiration. L'oeuvre de Christophe Dejours (2007, 2011, 2015, 2017) y occupant au Brésil une place historiquement centrale (Dejours, Abdoucheli, \& Jayet, 1994). Sa perspective psychodynamique du travail va en effet à partir du tournant des années quatre-vingt-dix irriguer très largement les recherches brésiliennes en psychologie du travail (Aerosa, 2019; Bendassolli \& Borges-Andrade, 2015; Bendassolli, 2010/2021; Betiol \& Tonelli, 2002; Cicero, Cardoso, \& Klipan, 2019; Lima, 1998; Sznelwar, Uchida, 
\& Lancman, 2011). Comme le soulignaient en 2009 dans un état des lieux de la psychodynamique du travail au Brésil, Álvaro Roberto Crespo Merlo \& Ana Magnólia Bezerra Mendes (2009):

Le lancement au Brésil du livre "Travail et usure mentale" (A loucura do trabalho), de Christophe Dejours, en 1987, grâce à son nouvel apport théorique, est devenu la référence pour quasiment tous les chercheurs du champ. La discussion et l'approche présentées dans ce livre étaient en effet nouvelles, ont apporté de nombreuses réponses et ouvert de nouvelles voies pour penser le travail, notamment sur son organisation et ses effets sur la santé psychique des travailleurs (p. 142). (Traduction de l'auteur)

Plus loin, ils ajoutaient:

L'approche adoptée par cette discipline a permis de dépasser une vision réductioniste qui responsabilisait uniquement les individus et de trouver des réponses opérationnelles spécifiques, qui s'inscrivaient dans les réalités concrètes du travail en mettant en évidence, par exemple, le rôle de l'intelligence des travailleurs et la fonction qu'elle jouait en tant que mécanisme de défense et de construction de l'identité au travail (Dejours, 2015, p. 143). (Traduction de l'auteur.)

En raison de son ancrage dans la pratique, cette approche va également exercer une influence notable dans les sciences administratives pour ceux et celles qui s'intéressent au rapport vie psychique-organisation (Fachin \& Cavedon, 2003; Freitas, 2008 a; Freitas \& Motta, 2000; Vergara, 2006), parfois via le chapitre qu'il avait rédigé dans mon ouvrage princeps (Dejours, 1996).

D'autres travaux dans le domaine des sciences du travail vont aussi susciter de l'intérêt. On peut penser ici à ceux de Pascale Molinier sur les emplois de service et de soins (Gaviria \& Molinier, 2011; Molinier, 2013), longtemps proche de Dejours, à ceux de Yves Clot (2010) et de Dominique Lhuillier, qui ont développé une clinique de l'activité du travail inspirée par Vitgovsky et Bakthine (Bendassolli \& Soboll, 2011/2021; Clot \& Lhuillier, 2006, 2010; Lima, 2013), et aux travaux pionniers menés en ergonomie par Alain Wisner, fondateur de l'Ecole française d'ergonomie (Sznelwar, 2006). Enfin, certains travaux de sociologie du travail ont eu également une certaine résonnance. On peut signaler en particulier la théorie de la régulation sociale de Jean-Daniel Reynaud (1989), une référence centrale dans le champ francophone (Terssac, 2003) et qui va connaitre un certain succès auprès de collègues en sciences administratives brésiliens, notamment auprès de Marlene Catarina Oliveira Lopes Melo (1984) qui, ayant fait sa thèse sous sa direction à Paris, va l'utiliser pour comprendre le rôle jouée par la participation dans la gestion des conflits au travail, un constat que l'on peut faire aussi de nos jours parmi les collègues de langue française (Richebé et al., 2020). Enfin, on peut mentionner les travaux de Norbert Alter sur l'innovation (2002) et la coopération (2010) qui n'ont pas manqué d'être remarqués par la communauté brésilienne (Salvador, 2011).

\section{Les sciences économiques hétérodoxes}

La troisième source à laquelle les chercheurs brésiliens en sciences administratives vont puiser, sont les sciences économiques hétérodoxes (Lévesque, Bourque, \& Forgues, 1997). En effet, tous ceux et toutes celles qui s'intéressent à l'équilibre socioéconomique, au développement social et aux organisations de l'économie sociale dans un pays où existent de profondes inégalités sociales, (Ferreira de Souza, 2018; IBGE, 2020), ont trouvé dans cer- 
tains courants de la pensée économique hétérodoxe française des éléments susceptibles d'enrichir leurs propres réflexions sur les réalités brésiliennes.

Parmi ces courants, on retrouve ceux qui tournent autour de la théorie de la régulation: Boyer, Freyssenet, Aglietta, Coriat, Lipietz, Orléan... (Aglietta, 1997; Boyer \& Cohen, 2010; Coriat, 1994; Mello, 2019), bien connue en Amérique latine (Castaingts-Teillery, 2014; de Mello Filho, 2019), et de l'économie des conventions (Boltanski \& Chiapello, 1999; Boltanski \& Thévenot, 1991; Eymard-Duvernay, 2004; Favereau, 1989; Orléan, 2004; Salais \& Thévenot, 1986), l'analyse des conventions étant une théorie de l'action collective, qui cherche à dépasser les contradictions de la théorie du choix rationnel (Bessy \& Favereau, 2003).

Les travaux de ce dernier courant, notamment ceux de Boltanski et Thévenot (1991) sur l'économie des grandeurs, portent en effet une attention centrale à la situation dans laquelle s'inscrit l'action collective. Celle-ci étant constituée, selon eux, de processus de justifications et d'épreuves; de ce processus surgit une pluralité de sens de ce qui est juste à travers plusieurs modèles de «mondes» (marchand, domestique, industriel, civique, de l'opinion, et de l'inspiration), sans qu'il existe une hiérarchie externe ou déterminée entre eux. L'existence de cette pluralité de conventions permet ainsi de critiquer une vision uniquement marchande de l'action. Ces travaux, en rejoignant les réflexions d'un certain nombre de chercheurs brésiliens autour de la rationalité substantive, dans la foulée du travail pionnier mené au Brésil par Guerreiro Ramos, vont avoir quelque résonnance auprès de ces derniers (Serva, Caitano, Santos, \& Siqueira, 2015).

L'économie sociale étant un important secteur au Brésil, les travaux menés dans l'univers francophone à ce sujet vont également susciter beaucoup d'intérêt chez nombre de chercheurs brésiliens tout comme les travaux brésiliens vont aussi susciter beaucoup d'intérêt en France et au Québec (Laville, 2011). La théorie des organisations ne s'étant pas trop intéressé jusqu'ici aux organisations de l'économie solidaire (Serva, 2002; Serva \&Andion, 2006), ce n'est donc pas surprenant, là encore, que certains chercheurs brésiliens vont aller puiser certaines inspirations dans ce courant de la sociologie économique, tout en y apportant une contribution originale (Andion, 1998, 2005; França, Laville, Medeiros, \& Magnen, 2005; Martes, Loureiro, Abramovay \& Serafim, 2007; Sperb \& Serva, 2018). Les colloques internationaux sur le pouvoir local, organisées à Bahia par le NEPOL, aujourd'hui le CIAGS et coordonnés par Tânia Fischer, en ayant favorisé leur diffusion.

L'ouvrage récent de Philippe Eynaud et de Genauto Carvalho de França de Filho (2019), membre du CIAGS, publié à la fois en France, Solidarité et organisation: penser une autre gestion, et au Brésil (2020), témoigne à sa façon de cette coopération franco-brésilienne exemplaire sur cette thématique fondamentale à la fois pour la gestion et notre avenir socioéconomique et environnemental. Ils en soulignent eux-mêmes d'ailleurs le potentiel mais aussi les paradoxes ainsi:

... Face à ces périls menaçants, une seule et même solution .... peut cependant être convoquée: la solidarité. La défense d'une solidarité entre les hommes et la nature (entendue comme l'ensemble des êtres vivants: animaux et végétaux) est en effet le moyen le plus direct de répondre aux défis du changement climatique et de la perte de la biodiversité. Le développement d'une solidarité entre les hommes eux-mêmes est aussi une réponse pérenne aux inégalités qui les divisent. Mais si l'énoncé de la réponse est facile, sa mise en œuvre est particulièrement ardue. Elle se confronte à de nombreux obstacles, parmi lesquels - et non le moindre - le déficit de réflexion sur les concepts mobilisables pour organiser cette solidarité au plus près des acteurs et de leur activité économique. Il y a en effet un paradoxe à sou- 
ligner d'emblée: la solidarité n’a jamais été aussi nécessaire à mettre en œuvre sur les plans national et international, et la réflexion à son propos n’a jamais été aussi éludée, méconnue, quand ce n'est pas discréditée (Eynaud et França, 2019, p. 5)

\section{ENTRE LOCALISME ET OUVERTURE: LE CHAMP BRÉSILIEN À LA RECHERCHE DE SA SINGULARITÉ DANS LE CHAMP INTERNATIONAL?}

Le champ des sciences administratives brésilien a toujours connu, comme nous venons de le voir, des influences étrangères, ce qui n'est pas totalement surprenant dans le monde scientifique marqué traditionnellement par la circulation des idées. Si ces échanges peuvent se montrer très fructueux, il reste que certaines situations peuvent être problématiques quand une forte domination s'exerce sur un champ particulier, l'empêchant de se développer à son rythme et selon ses propres questionnements. C'est le cas aujourd'hui de nombreux champs nationaux, qui de plus en plus sous la pression de règles et de logiques dites "internationales", mises en place dans les faits au sein de l'univers nord-américain, tentent de s'y conformer. Ce phénomène n'est d'ailleurs pas uniquement vécu dans les pays du Sud, il touche également de nombreux autres pays dans le monde y compris anglophones (Clegg \& Bayley, 2007; Dameron \& Durand, 2017; Willmott, 2011).

Dans l'univers de l'enseignement et de la recherche en gestion, il se caractérise entre autres par ces nombreux classements annuels, internationaux et nationaux, d'institutions (Shanghai, Times, Financial Times...), lesquels se fondent à la fois sur les accréditations internationales obtenues (AACSB, EQUIS, AMBA), le nombre de publications du personnel enseignant qui apparaissent dans des revues classées, les plus cotées étant toujours américaines, et bien sûr par l'hégémonie croissante de la langue anglaise dans le champ que cela implique (Chanlat, 2014 a, b et c; Collectif, 2019; Dameron \& Durand, 2017; Lussier \& Chanlat, 2017; Nygaard, 2019; Tietze \& Dick, 2012; Tsuda, 2013). Ces classements bibliométriques étant de plus en plus critiqués (Berry, 2009; Willmott, 2011; Tourish \& Willmott, 2015), certaines institutions n'hésitent pas à s'en détacher, comme vient de le faire dernièrement en France la section Gestion-Economie du CNRS, en supprimant définitivement le sien.

Malgré tout, pour les pays non-anglophones, la tension demeure forte entre adopter une stratégie dite "internationale", qui se confond souvent avec "le tout en anglais", et donc une anglicisation croissante des esprits (Boussebaa \& Tienari, 2019; Chanlat, 2014a; Collectif, 2019; Jackson \& Primecz, 2019; Lussier \& Chanlat, 2017; Nygaard, 2019; Tietze \& Dick, 2012; Tsuda, 2013), et une stratégie qui vise à demeurer pertinente pour son environnement local, régional et national, tout en évitant la fermeture sur soi.

Dans le cas des pays latino-américains et du Brésil en particulier, ces questions font, comme nous l'avons vu, régulièrement l'objet de nombreux débats au sein de la communauté scientifique (Alcadipani \& Caldas, 2012; Alcadipani \& Faria, 2014; Bertero, Caldas, \& Wood, 1999; Guedes \& Faria, 2010; Ibarra-Colado, Faria \& Guedes, 2010; Ibarra-Colado, 2006; Paula et al., 2010; Serva, 2017). Face à cette situation, si certains semblent succomber aux règles dominantes, d'autres tentent toutefois d'y résister. Au Brésil, cette résistance passe par une critique des logiques dominantes (Bertero et al., 2013; Fischer, Waiandt, \& Fonseca, 2011), des systèmes de classement (Fischer, 2018; Serva, 2017), de la politique du tout anglais en matière de publication (Alves \& Pozzebon, 2013; Gantman, Yousfi, \& Alcadipani, 2015) et par l'affirmation de ses singularités (Motta, 1983, 2002; Motta \& Caldas,1997; Fachin, 2006, 2014; Davel, Vergara \& Ghadiri, 2007; Waiandt, 2009, 2018) et une ré-interrogation des travaux faits dans les 
pays du Sud à partir d'une réflexion postcoloniale et d'une situation sociale profondément inégalitaire (Alcadipani, 2010b; Alcadipani \& Faria, 2014; Alcadipani \& Rosa, 2011; Gantman et al., 2015; Ferreira de Souza, 2018; IBGE, 2020). Ces critiques prônent en effet une refonte du système dans son ensemble:

... Face au pouvoir impérial d'un "réoccidentalisme" et à l'obligation d'assumer des responsabilités géoépistémiques plus substantielles, un des deux principaux défis au Brésil pour les prochaines décennies sera de reconstruire et réformer les institutions existantes (Anpad, Capes, CNPq etc.) ... De cette manière, le champ brésilien de la gestion sera en mesure de mener des transformations du même type, que celles entreprises par d'autres institutions extérieures (US Academy of Management, Chinese Academy of Management, Banco Mundial, Egos etc.) à travers un grand projet qui embrasserrait une géopolitique de la connaissance, et promouvrait la substitution d'un universalisme eurocentrique au profit d'une vision pluraliste du monde permettant ainsi de construire un champ scientifique au sein duquel une diversité d'univers et de savoirs pourrait coexister. Si un tel projet peut demander du temps, ou encore trop exiger du champ de la gestion, cela pourrait constituer néanmoins une excellente occasion de le réinventer ... (Bertero et al., 2013, p. 190). (Traduction de l'auteur.)

Elles mettent en garde contre une évaluation uniquement bibliométrique de la recherche qui laisse la part du lion aux publications de langue anglaise et contribue à réduire la créativité:

Nous devons faire très attention à ce que les valeurs et les paramètres qui nous régulent n'aboutissent pas à la castration intellectuelle que nous ne méritons pas mais bien à encourager les efforts créatifs et innovants. La question principale est en effet ici: quel est l'impact que ma gestion a sur les personnes, les territoires et les organisations? Par impact, nous entendons l'évaluation de ce qui s'aggrège, en évitant ce quasi-autisme que l'on retrouve chez les chercheurs qui s'isolent et ne se laissent guider que par des mesures métriques. En effet, dans quelques années ces paramètres pourraient se révéler obsolètes. Il vaut mieux être plus attentif dès maintenant à cette question de la pertinence de nos recherches pour les univers qui nous environnent, ce qui permettrait à notre champ de se repositionner en conséquence (Fischer, 2018, p.26). (Traduction de l'auteur.)

Au Brésil, l'agence de régulation CAPES a mis en place son propre classement des revues: le classement Qualis. C'est la principale échelle de référence pour l'évaluation des programmes d'études supérieures, sur la base des publications des professeurs de ces programmes dans les revues les mieux positionnées dans ce classement. La construction de cette échelle Qualis reflète un éventail d'indices et de facteurs d'impact qui sont considérées par les sociétés scientifiques concernées. Dans le champ de l'administration, dans la strate supérieure ( $\left.A_{1}\right)$ du classement Qualis, il n'y a aucune revue brésilienne; dans la grande majorité des cas, ce ne sont que des revues américaines qui s'y trouvent, la langue anglaise étant totalement prédominante dans ce classement (Serva, 2017b, p. 55). (Traduction de l'auteur.) 
Enfin, elles réaffirment l'importance du dialogue avec d'autres champs, et le renforcement des coopérations internationales en vue de faire face aux redoutables défis qui se posent à nos sociétés et à notre planète (Alcadipani \& Faria, 2018; Chanlat, Fachin, \& Fischer, 2006, 2007; Eynaud \& França, 2019). Pour nous, c'est dans ce cadre que le dialogue entre les chercheurs de langue française et les chercheurs brésiliens doit continuer à se faire.

C'est dans ce contexte, comme nous venons de le voir, que les travaux de langue française ont trouvé depuis quelques décennies un écho certain au sein de la communauté brésilienne, notamment à travers quelques oeuvres et courants marquants. Cet intérêt a reposé sur un atout historique, et une proximité culturelle latine, qui, en dépit des différences qu'il peut y avoir entre les Latins eux-mêmes, en l'occurrence ici, les Français et les Brésiliens, existe malgré tout (Pinot de Villechenon, 2003; Rouquié, 1987; Pinot de Villechenon, Chanlat, \& Rizzo, 2021).

Ce dialogue ne pourra toutefois perdurer, selon moi, que si les chercheurs de langue française, tout comme les chercheurs brésiliens, continuent à défendre leurs spécificités, à rédiger dans leur langue, livres, articles et monographies, à organiser des colloques et des séminaires où les questions propres à leur société soient toujours prises en compte (Chanlat, 2014a, 2014b); et qu'ils ou elles puissent ainsi continuer à s'adresser à leur environnement socioéconomique de manière pertinente (Fischer, 2018). Il faut en effet éviter de se retrouver dans la situation de ce chercheur norvégien, qui avouait être incapable désormais de présenter le résultat de ses études à ses concitoyens en norvégien, en raison de la politique du tout anglais mis de l'avant par son institution qui lui en avait fait perdre la capacité (Nygaard, 2019).

Pour ma part, comme je viens de le souligner, j'ai essayé, au cours de toutes ces années, d'être un acteur dans l'affirmation de ces singularités locales, régionales et nationales, dans tous les espaces où je suis intervenu (enseignement, recherche, publications, associations scientifiques nationales et internationales), attaché que je suis depuis toujours à un véritable dialogue interculturel (Chanlat, 2014b; Chanlat, Davel, \& Dupuis, 2013; Chanlat \& Pierre, 2018; Davel, Dupuis \& Chanlat, 2008) entre univers linguistiques, et à rester, notamment attentif à ce lien avec la communauté brésilienne qui m'est chère pour les raisons que j'ai soulignées plus haut, et qui possède, selon moi, malgré toutes les critiques émises précédemment, tous les éléments d'un champ scientifique, aspirant à une relative autonomie (le nombre, les revues, les maisons d'édition, les associations scientifiques, les congrès,..) (Chanlat, 2014b, 2015). C'est la raison pour laquelle j'ai encouragé le plus souvent possible de nombreux collègues, notamment de langue française, à participer à des évènements au Brésil et de nombreux collègues brésiliens à participer à des événements de langue française et que j'ai essayé de diffuser certains de leurs travaux en français, y compris dans la collection que je dirige aux Presses de l'Université Laval (Calvacanti, 2011).

Cet esprit d'ouverture repose par ailleurs sur un certain plurilinguisme et une politique active en matière de traduction qui permette d'avoir accès à des pensées étrangères (Initiative d'Helsinki, 2019). Ce qui n'est bien souvent pas le cas de nos collègues américains dont le monolinguisme et le peu de traductions disponibles de nos travaux de référence en anglais, les conduit trop souvent à un provincialisme académique, comme le rappelle Gaylord Georges Candler (2014) à propos de la réception de la pensée de Guerreiro Ramos aux Etats-Unis; le provincialisme n'étant pas bien sûr uniquement l'apanage de nos collègues américains. Les chercheurs brésiliens, en maitrisant souvent plusieurs langues, sont donc bien placés pour éviter cet écueil si ce plurilinguisme est utilisé pour développer des travaux originaux sur leur réalité tout en dialoguant avec d'autres univers linguistiques en connaissance de cause (Clegg, \& Bayley, 2007; Bertero et al., 2013; Chanlat, 2014a, 2015) et concrétiser ainsi cette pensée de Guerreiro Ramos (1981/1989, p.5): " Ce n'est qu'à travers l'expérience libre de la réalité et de son articulation précise que l'on peut comprendre la rationalité substantive". (Notre traduction). 
A l'occasion de ce numéro spécial de la $R A E$, je tiens à conclure cet article par un souhait qui va dans ce sens: que ces relations entre chercheurs brésiliens et francophones se maintiennent et se développent dans une réciprocité bienveillante, comme le souhaitait Guerreiro Ramos (1984). Cela se fera par la présence régulière de travaux publiés dans nos différentes revues et nos divers éditeurs, et bien sûr, dans cette revue de référence multilingue qu'est devenue la RAE (Tonelli, 2018), à qui je souhaite pour l'occasion tous mes meilleurs vœux dans la continuation de cette formidable aventure, commencée il y a maintenant soixante ans. Soixante ans, c'est en effet tout un anniversaire pour une revue scientifique dans le champ des sciences administratives.*

* Pour rappel, la revue américaine Administrative Science Quarterly a été fondée en 1956, juste cinq ans avant.

\section{NOTE DE L'AUTEUR}

Professeur émérite, Université Paris-Dauphine PSL, Dauphine Recherches en Management, Paris, France.

Professeur invité, Institut Mines-Télécom Business School, Paris, France.

Professeur affilié, HEC-Montréal, Départment of Management, Montréal, Canada.

\section{REFERENCES}

Aerosa, J. (2019). 0 mundo do trabalho em (re)análise: Um olhar a partir da psicodinâmica do trabalho. Laboreal, 15(2), 1-24. doi: $10.4000 /$ laboreal.15504

Aglietta, M. (1997). Régulation et crises du capitalisme. Paris, France: Odile Jacob.

Aktouf, O. (1996). A administração entre tradição e a renovação. São Paulo, SP: Casa Editorial Atlas.

Alcadipani, R. (2010a). Critical international management and international critical management: Perspectives from Latin America. Critical Perspectives on International Business, 6, (2/3), 86-96. doi: 10.1108/cpoib.2010.29006baa.001

Alcadipani, R. (2010b). From Latin America to the world: Notes on the (possible) latin american management styles. In A. Guedes, \& A. Faria (Eds.), International management and international relations: A critical perspective from Latin America (Vol. 1, pp. 136-158). New York, USA: Routledge.

Alcadipani, R., \& Bertero, C. (2012). Guerra Fria e ensino do management no Brasil: 0 caso da FGV/EAESP. RAE-Revista de Administração de Empresas, 52(3), 284-299. doi: 10.1590/ S0034-75902012000300002

Alacadipani, R., \& Bertero, C. O. (2014l). Uma escola NorteAmericana Ultramar? Uma historiagrafia da EAESP. RAERevista de Administração de Empresas, 54(2), 154-169. doi: /10.1590/So034-759020140204

Alcadipani, R., \& Caldas, M. (2012). Americanizing Brazilian management. Critical Perspectives on International Business, 8(1), 37-55. doi: 10.1108/17422041211197558
Alcadipani, R., \& Cooke, B. (2013). The Ford Foundation's mess in Brazil. Best Paper Proceedings of the Academy of Management Meeting. doi: 10.5465/ambpp.2013.218

Alcadipani, E., \& Faria, A. (2014). Fighting Latin American marginality in "International business". Critical Perspectives on International Business, 10(1/2), 107-111. doi: 10.1108/ cpoib-11-2013-0047

Alcadipani, R., \& Rosa, A. R. (2011). From global management to glocal management: Latin American perspectives as a counter-dominant management epistemology. Canadian Journal of Administrative Science, 28(4), 453-466. doi. org/10.1002/cjas.165

Alter, N. (2002). L'innovation ordinaire. Paris, France: PUF.

Alter, N. (2010). Donner et prendre: La coopération en entreprise. Paris, France: La Découverte.

Alves, M. A., \& Pozzebon, M. (2013). How to resist linguistic domination and promote knowledge diversity. RAE-Revista de Administração de Empresas, 53(6), 629-633. doi: 10.1590/ So034-759020130610

Amado, G. (2013). Les processus psychiques au sein des groupes de travail: au-delà de Bion et de Pichon-Rivière. Nouvelle Revue de Psychosociologie, 1(15), 163-182. Récupéré de https://www.cairn.info/revue-nouvelle-revuede-psychosociologie-2013-1-page-163.htm

Amblard, H., Bernoux, P., Herreros, G., \& Livian, Y.-F. (1995). Les nouvelles approches en sociologie des organisations. Paris, France: Seuil. 
Andion, M.-C. (1998). Gestão em organizações da economia solidária: Contornos de uma problemática. Revista de Administração Pública, 32(1), 7-25. Récupéré de http://bibliotecadigital. fgv.br/ojs/index.php/rap/article/view/7680

Andion, M.-C. (2005). A gestão no campo da economia solidária: Particularidades e desafios. Revista de Administração Contemporânea, 9, 79-99. doi: 10.1590/S141565552005000100005

Andion, C., \& Serva, M. A. (2006). Etnografia e os estudos organizacionais. In K, C, Godoi., R, Bandeira-de-Mello, \& A., Barbosa da Silva. (Orgs.), Pesquisa qualitativa em estudos organizacionais: Paradigmas, estratégias e métodos. São Paulo, SP: Saraiva.

Araújo, N. G. de, \& Barros, V. A. de. (2019). A psicologia do trabalho e as clínicas do trabalho no Brasil. Laboreal, 15(2), 1-12. doi: 10.400o/laboreal.15515

Aubert, N., \& Gauléjac, V. de. (1993). Coste de la excelenciai. Madrid, España: Paidos.

Audet, M., \& Déry, R. (1996). La science réfléchie: Quelques empreintes de l'épistémologie des sciences de l'administration. Anthropologie et Sociétés, 20, 1, 103-123. doi : $10.7202 / 015397$

Audet, M., \& Malouin, J.-L. (Orgs.). (1986). La production des connaissances scientifiques de l'administration. Québec, Canada: Les Presses de l'Université Laval.

Azevedo, A., \& Albernaz, R. (2006). A "antropologia" do guerreiro: A história do conceito de homem parentético. Em memória a Eliana Guerreiro Ramos (1949-2003). Cadernos EBAPE.BR, 4(3), 1-19. Récupéré de http://bibliotecadigital.fgv. $\mathrm{br} / \mathrm{ojs} /$ index.php/cadernosebape/article/view/7398

Barreto, O. (1999). Escola de Administração da UFBA: 40 anos de história. Organizações e Sociedade, 6(15), 9-14. doi: 10.159o/ S1984-92301999000200001

Barros, A., Alcadipani, R., \& Bertero, C. O. (2018). A criação do curso superior em administração na ufrgs em 1963: uma análise histórica [The creation of the higher education course in administration at UFRGS in 1963: A historical analysis]. RAE-Revista de Administração de Empresas, 58(1), 3-15. doi: 10.1590/s0034-759020180102

Bartel-Radic, A. (2013). 'Estrangeirismo' and flexibility: intercultural learning in Brazilian. MNCs. Management International/International Management/ Gestión International, 17(4), 239-253. doi: 10.7202/1020680

Barus-Michel, J., Enriquez, E., \& Lévy, A. (Dir.). (2002). Vocabulaire de Psychosociologie. Ramonville Saint-Agne, France: Éditions Érès

Bayart, D., Borzeix, A., \& Dumez, H. (Dir.), (2010). Langage et organisations: Sur les traces de Jacques Girin. Palaiseau, France: Presses de l’École Polytechnique.

Bélanger, P., \& Lévesque, B. (1996). La modernisation sociale des entreprises. Montréal, Canada: Presses de l'Université de Montréal.
Bendassolli, P., \& Borges-Andrade, J. (Orgs.). (2015). Dicionário de psicologia do trabalho e das organizações. São Paulo, SP: Artesa.

Bendassolli, P., \& Soboll, L. A. P. (Orgs.). (2011/2021). Clínicas do trabalho, novas perspectivas para compreender do trabalho na atualidade. São Paulo, SP: Atlas.

Bernoux, P (1985). Sociologie des organisations. Paris, France: Seuil.

Berry, M. (1983). La technologie invisible. Paris, France: Ecole Polytechnique.

Berry, M. (2009). Les mirages de la bibliométrie, ou comment scléroser la recherche en croyant bien faire. Revue du MAUSS, 1(33), 227-245. Récupéré de https://www.cairn.info/revuedu-mauss-2009-1-page-227.htm

Berry, M. (2011). Savoirs théoriques et gestion. In J.-M. Barbier (Dir.), Savoirs théoriques et savoirs d'action (pp. 43-56). Paris, France: Presses Universitaires de France.

Bertero, C. O. (2006). Ensino e pesquisa em administração. São Paulo, SP: Thomson Learning.

Bertero, C. O., Alcadipani, R., Cabral, S., Faria, A., \& Rossoni, P. (2013). Os desafios da produção de conhecimento em administração no Brasil. Cadernos EBAPE.BR, 11(1), 181-196.

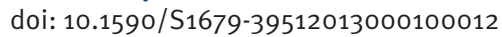

Bertero, C. O., Caldas, M. P., \& Wood, T., Jr. (1999). Produção científica em administração: Provocações, insinuações e contribuições para o debate local. Revista de Administração Contemporânea, 3(1), 147-178. doi: 10.1590/S1415 65551999000100009

Bertero, C. O., Caldas, P. P., \& Wood, T. (Coord.). (2005). Produção científica em administração no Brasil: $O$ estado-da-arte. São Paulo, SP: Atlas.

Bessy, C., \& Favereau, O. (2003). Institutions et économie des conventions. Cahiers d'Économie Politique, 1(44), 119164. doi: 10.3917/cep.044.0119

Betiol, M. I. S., \& Tonelli, M. J. (2002). A Trama drama numa intervenção: Análise sobra oticada psicodinâmica do trabalho. Organizações \& Sociedade, 9(24), 11-23. doi: 10.1590/S198492302002000200001

Boltanski, L., \& Chiapello, E. (1999). Le nouvel esprit du capitalisme. Paris, France: Gallimard.

Boltanski, L., \& Thévenot, L. (1991). De la justification: Les économies de la grandeur. Paris, France: Gallimard.

Bonnafous, E. (1953). Les liens intellectuels entre la France et l'Amérique latine. Revue Des Deux Mondes (1829-1971), 6386. Récupéré de http://www.jstor.org/stable/44585861

Boussebaa, M., \& Tienari, J. (2019). Englishization and the politics of knowledge production in management studies. Journal of Management Inquiry, 30(1), 59-67. doi: $10.1177 / 1056492619835314$

Bouville, G., \& Yousfi, H. (Dir.). (2021). Les dimensions oubliées de la gestion, anthropologie, management, travail et organisation: Mélanges en l'honneur du professeur JeanFrançois Chanlat. Ste-Foy, Québec, Canada: Les Presses de l'Université Laval; Paris, France: Hermann. 
Boyer, R., \& Cohen, P. (2010). Teoria da regulação: Os fundamentos. São Paulo: Estação Liberdade

Buarque de Holanda, S. (1936). Raízes do Brasil. Rio do Janeiro: Editora José Olympio Editora. Edição francesa, (1998) Racines du Brésil, Paris: UNESCO/Gallimard.

Bueno, J. M., \& Freitas, M. E. (2016). Les équipes multiculturelles dans les filiales brésiliennes de multinationales. In J.-P. Dupuis, J.-P. Lemaire, \& E. Milliot (Coord.), Ancrages culturels dans un monde en mutation (pp. 157-172). Paris, France: Vuibert.

Caldas, M., Fachin, R., \& Fischer, T. (1999). Prefácio à edição brasileira do Handbook de Estudos Organizacionais. Vol.1: Modelos de Análise e Novas Questões em Estudos Organizacionais. (Eds), Clegg, S., Hardy, C., \& Nord, W. São Paulo, SP: Atlas, 61-98.

Caldas, M. P., \& Wood, T., Jr. (1997). For the English to see': The importation of managerial technology in late 2oth-century Brazil. Organization, 4(4), 517-534. doi: 10.1177/135050849700400410

Calvacanti, B. (2011). Le leadership dans les organisations publiques au Brésil. Ste-Foy, Québec, Canada: Les Presses de l'Université Laval.

Candler, G. G. (2014). Linguistic diglossia and parochialism in American public administration: The missing half of Guerreiro Ramos's redução sociológica.Administrative Theoryand Praxis, 28(4), 540-561. doi: 10.1080/10841806.2006.11029561

Cassundé, F. R., Barbosa, M. A. C., \& Mendonça, J. R. C. de. (2016). A influência da tradição anglo-saxônicas estudos organizacionais brasileiros: 0 que mudou (ou não) nos últimos 15 anos? Perspectivas em Gestão \& Conhecimento, 6(1), 238-254. Récupéré de https://periodicos.ufpb.br/ojs2/ index.php/pgc/article/view/27064

Castaingts-Teillery, J. (2014). La crisis financiera. Un análisis de etnoeconomía. Conferencia, Academia Mexicana de Economía Política, Ciudad de México, México. Récupéré de amepmexico.com.mx.

Chanlat, A., \& Dufour, M (dir) (1985). La rupture entre l'entreprise et les hommes. Montréal, Québec, Canada: Québec/ Amériques.

Chanlat, J.-F. (Dir.). (1992a). Indivíduo na organização: Dimensões esquecidas (Vol. I). São Paulo, SP: Publicação Atlas, Montréal, Québec, Canada: HEC CETAI.

Chanlat J.-F. (1992b). L'analyse des organisations: Un regard sur les productions de langue française contemporaines (19501990). Cahiers De Recherche Sociologique, (18-19), 93-138. doi: $10.7202 / 1002305$ ar

Chanlat, J.-F. (Dir.). (1994a). O indivíduo na organização: Dimensões esquecidas (Vol. II). São Paulo, SP: Publicação Atlas, Montréal, Québec, Canada: HEC-CETAI.

Chanlat, J.-F. (1994b). Francophone organizational analysis (1950-1990): An overview. Organization Studies, 15(1), 47-79. doi: 10.1177/017084069401500103

Chanlat, J.-F. (Dir.). (1996). O indivíduo na organização: Dimensões esquecidas (Vol. III), São Paulo, SP: Publicação Atlas, Montréal, Québec, Canada: HEC CETAI.
Chanlat J.-F. (2000). Ciências sociais e administração. São Paulo, SP: Publicação Atlas.

Chanlat, J.-F. (2010a). Gestão das empresas: Uma perspectiva antropológica. (Coleção Debates em Administração). São Paulo, SP: Cengage Learning.

Chanlat, J.-F. (2010b). Hommage à José Roberto Gomes da Silva. Revue Française de Gestion, 36(20), 183-184. Récupéré de https://www.cairn.info/revue-francaise-de-gestion-20104-page-183.htm

Chanlat, J.-F. (2014a). Langue et pensée dans la recherche en gestion: Constats, enjeux et atouts de la langue française. Gérer et Comprendre, 115(1), 4-17. doi: 10.3917/geco.115.0004

Chanlat, J.-F. (2014b). A língua e o pensar no campo da pesquisa em administração: Constatações e questões para os pesquisadores que não utilizam a língua inglesa. RAE-Revista de Administração de Empresas, 54(6), 706-714. doi: 10.159o/ So034-759020140610

Chanlat, J.-F. (2014C). Language and thinking in organization studies: The visibility of French OS production in the AngloSaxon OS field. International Journal of Organizational Analysis, 22 (4), 504-533. doi: 10.1108/IJOA-09-2013-0708

Chanlat J.-F. (2015). Le champ des études organisationnelles: Le regard critique d'un chercheur plurilingue. RAE-Revista de Administração de Empresas, 55(2), 226-230. doi: 10.1590/ So034-759020150212

Chanlat, J-F. (2020). La catastrophe sanitaire actuelle: un fait social total. Le Libellio d'AEGIS, 16(7), p. 3-30. Récupéré de https://hal.archives-ouvertes.fr/hal-03120567

Chanlat, J.-F, Davel, E., \& Dupuis, J.-P. (2013). Cross-Cultural Management. Culture and management across the world. Londres, RU: Routledge.

Chanlat, J.-F, Dameron, S., Dupuis, J.-P., Freitas, M,E, \& Özbilgin, M. (2013). Management et Diversité : lignes de tension et perspectives. Management international / International Management / Gestiòn Internacional, 17, 5-13. doi: 10.7202/1015807ar

Chanlat, J.-F., Özbilgin, M. (2018). Management et diversité: Comparaisons internationales. Ste-Foy, Québec, Canada: Les Presses de l'Université Laval, Paris, France: Hermann.

Chanlat, J.-F., Özbilgin, M. (2018). Management et diversité: approches thématiques et défis sociopolitiques. Ste-Foy, Québec, Canada: Les Presses de l'Université Laval, Paris, France: Hermann.

Chanlat, J.-F., Pierre, P.(2018). Management interculturel. Evolution, tendances et critiques. Caen, France: EMS.

Chanlat, J.-F., Fachin, R., \& Fischer, T. (Dir.). (2006). Analyse das organizações: Perspectivas latinas (Vol. 1). Porto Alegre, Brasil: UFRGS

Chanlat, J.-F., Fachin, R., \& Fischer, T. (Dir.). (2007). Analyse das organizações: Perspectivas latinas (Vol. 1). Porto Alegre, RS: UFRGS

Chanlat, J-F.,\& Séguin, F. (1987). L'analyse des organisations. Tome II: les composantes de l'organisation, Montréal, Québec, Canada: Gaëtan Morin. 
Cicero, A. F. C., Cardoso, J. M. M., \& Klipan, M. L. (2019). Panorama da psicodinâmica do trabalho no Brasil entre os anos de 2005 e 2015. Revista Interinstitucional de Psicologia, 12(1), 19-36. doi: $10.36298 /$ gerais 2019120103

Clarke, T., O'Brien, J., \& O'Kelley, C. (2019). The Oxford Handbook of The Corporation. Oxford, UK: Oxford University Press.

Clegg, S R., \& Bayley, J.R. (Eds). (2007). International Encyclopedia of Organization studies. Thousand. Oaks: Sage.

Clegg, S., Hardy C., \& Nord, W. (Ed.). (1999). Handbook de Estudos Organizacionais: Modelos de Análise e Novas Questões em Estudos Organizacionais (Vol.1). São Paulo, SP: Atlas.

Clegg, S., Hardy C., \& Nord, W. (Ed.). (2001). Handbook de Estudos Organizacionais: Reflexões e Novas Direções (Vol.2). São Paulo, SP: Atlas.

Clegg, S., Hardy C., \& Nord, W. (Ed.). (2004). Handbook de Estudos Organizacionais: Ação e Análise Organizacionais. (Vol. 3), São Paulo, SP: Atlas.

Clot, Y. (2010). Trabalho e poder de agir. Belo Horizonte, MG: Fabrefactum.

Clot, Y., \& Lhuillier, D. (2006). Perspectives en cliniques du travail. Toulouse, France: Erès.

Clot, Y., \& Lhuillier, D. (2010). Agir en clinique du travail. Toulouse, France: Erès.

Collectif. (2019, Février). La recherche francophone en sciences de gestion n'a aucune raison d'acccepter une soumission à un ordre anglo-saxon. Le Monde, 20. Récupéré de https://www. lemonde.fr/idees/article/2019/02/20/la-recherche-francophone-en-sciences-de-gestion-n-a-aucune-raison-d-accepterune-soumission-a-un-ordre-anglo-saxon_5425600_3232.html

Coriat, B. (1994). Pensar pelo avesso. Rio de Janeiro, RJ: UFRJ/ Revan.

Crozier, M. (1963a). Subdesenvolvimento, administração e burocracia. RAE-Revista de Administração de Empresas, 3(9), 117-134. Récupéré de http://bibliotecadigital.fgv.br/ojs/ index.php/rae/article/view/41005

Crozier, M. (1963b). Le phénomène bureaucratique. Paris, France: Seuil.

Crozier, M. (1981). O fenômeno burocrático: Ensaio sobre as tendências burocráticas dos sistemas de organização modernos e suas relações na França, com o sistema social e cultural (Vol. 2.). (J. A. Gil Sobrinho, Trad.). Brasília, DF: Editora Universidade de Brasília.

Crozier, M. (2000). À quoi sert la sociologie des organisations? Paris, France: Arslan.

Crozier, M., \& Friedberg, E. (1977). L'acteur et le système. Paris, France: Seuil.

Dameron, S., Durand, T. (Eds). (2017). The Future of Management Education. Volume 1: Challenges facing Business Schools around the World. London, UK: Palgrave.

Davel, E., Dantas, M., \& Vergara, S. C. (2008). Culture et gestion au Brésil. In E. Davel, J.-P. Dupuis, \& J.-F. Chanlat (Eds.), Gestion en contexte interculturel. Québec, Canada: Presses de l'Université de Laval et Téléuniversité. CD.
Davel, E., \& Dupuis, J.-P., \& Chanlat, J.-F. (Eds.). (2008). Gestion en contexte interculturel. Québec, Canada: Presses de l'Université de Laval et Téléuniversité.

Davel, E., \& Vergara, S, C. (Orgs.). (2001). Gestão com pessoas e subjetividade. São Paulo, SP: Atlas.

Davel, E., Vergara, S., \& Ghadiri, S. (Orgs.). (2007). Administração com arte: Experiências vividas de ensino-aprendizagem. São Paulo, SP: Atlas.

Davel, E. P. B., \& Melo, M. C. de O. L. (2005). Gerência em ação: Singularidades e dilemas do trabalho gerencial. Rio de Janeiro, RJ: Editora FGV.

David, A., Hatchuel, A., \& Laufer, R. (2012). Les nouvelles fondation des sciences de gestion. Paris, France: Presses des Mines.

Dejours, C. (1996). Uma nova visão do sofrimento humano nas organizações. In J.-F. Chanlat (Dir.), O indivíduo na organização: Dimensões esquecidas. (Vol 1). São Paulo, SP: Editora Atlas, 149-174.

Dejours, C. (2007). Fator humano. São Paulo, SP: Editora Atlas.

Dejours, C. (2011). A banalização da injustiça social. São Paulo, SP: Editora Atlas.

Dejours, C. (2015). A loucura do trabalho: Estudo de psicopatologia do trabalho. São Paulo, SP: Cortez EditoraOboré.

Dejours, C. (2017). Psicodinâmica do trabalho: Casos clínicos. São Paulo, SP: Dublinense.

Dejours, C., Abdoucheli, E., \& Jayet, C. (1994). Psicodinâmica do trabalho. São Paulo, SP: Atlas.

d'Iribarne, P., Chevrier, S., Henry, A., Segal, J.-P., \& Tréguer-Felten, G. (2020). Cross-cultural management revisited: A qualitative approach. Oxford, UK: Oxford University Press.

d'Iribarne, P., Henry, A., Segal, J.-P., Chevrier, S., \& Globokar, T. (1998). Cultures et mondialisation: Gérer par-delà les frontières. Paris, France: Seuil.

Dufour, M.,Chanlat,A.(1985). La rupture entre l'entreprise et les hommes. Montréal, Québec, Canada: Québec/Amériques.

Dumez, H. (2016). Méthodologie qualitative: Les questions clés de la démarche compréhensive. Paris, France: Vuibert.

Dupuis, J.-P., \& Kuzminski, A. (1998). Sociologie de l'économie, du travail et de l'entreprise. Montréal, Canada: Gaëtan Morin éditeur.

Enriquez, E. (1990). Da horda ao estado: Psicanálise do vínculo social. São Paulo, SP: Editora Senac.

Enriquez, E. (1997). A organização em análise. Capa comun. Kindle Direct Publishing.

Enriquez, E. (2007). As figuras do poder. Capa Comun, Kindle Direct Publishing.

Enriquez, E. (2014). Jogos de poder na empresa: Sobre os processos de poder e estrutura. São Paulo, SP: Zagodoni Editora. 
Entreprises et Histoire. (2016). Cinquante ans de sociologie des organisations. [Numéro spécial], 3(84). Paris, FR: ESKA

Etzioni, A. (1967). Organizações complexas. São Paulo, SP: Pioneira.

Eymard-Duvernay, F. (2004). Economie politique de l'entreprise. Paris, France: La découverte

Eynaud, P., \& França, C. de., Filho. (2019). Solidariedade e organizações: Pensar uma outra gestão. São Paulo, SP: Ateliê de Humanidades Editorial. [Edition française, Solidarité et organisation: Penser une autre gestion. Toulouse, France: Erès.

Fachin, R. (2006). Construindo uma associação científica: Trinta anos da ANPAD - Memórias, registros, desafios. Porto Alegre, RS: ANPAD.

Fachin, R. (2014). Memórias e posicionamentos em estudos organizacionais. Revista Brasileira de Estudos Organizacionais, 1(1), 4-16. doi: 10.21583/2447-4851.rbeo.2014.v1n1.27V

Fachin, R., \& Cavedon, N. R. (2003). Em busca da especificidade da influência francesa na análise organizacional no Brasil. Cadernos EBAPE.BR, 1(1), 1-13. doi: 10.1590/S167939512003000100006

Favereau, O. (1989). Organisation et marché. Revue française d'économie, 4(1), 65-96. doi: 10.3406/rfeco.1989.1203

Ferreira de Souza, P, H, D. (2018). Uma história de desigualdade: a concentração de renda entre os ricos no Brasil, 1926-2013. São Paulo, SP: Hucitec.

Fischer, T., \& Davel, E. (2018). Administração como aprendizagem: do vir ao porvir com Tânia Fischer. Farol Revista de Estudos Organizacionais e Sociedade, 5(12), 13-31. Récupéré de https://revistas.face.ufmg.br/index.php/farol/ article/view/4794

Fischer, T., Caldas, M., \& Fachin, R. (Orgs.). (2004a). Handbook de estudos organizacionais: Ação e análises organizacionais (Vol. 2). São Paulo, SP: Editora Atlas.

Fischer, T., Caldas, M., \& Fachin, R. (Orgs.). (2004b). Handbook de estudos organizacionais: Ação e análises organizacionais (Vol. 3). São Paulo, SP: Editora Atlas.

Fischer, T., Waiandt, C., \& Fonseca, R. L. (2011). A história do ensino em administração: Contribuições teóricometodológicas e uma proposta de agenda de pesquisa. Revista de Administração Pública, 45(4), 911-939. doi: 10.1590/So034-76122011000400002

Fischer, T. M. (1985). 0 ensino de administração pública no Brasil: Da tutela estrangeira à autonomia necessária. In Reunião Nacional da ANPAD (pp165-170). Anais... Florianópolis: Ed. UFSC

França, G, C. dé, Filho, Laville, J.-L., Medeiros, A. J. de S., \& Magnen, J.-L. (2005). Ação pública e economia solidária: Uma perspectiva internacional. Salvador, Bahia: Editora CIAGS/UFBA.

Freitas, M. E. (1991). Cultura organizacional: Grandes temas em debates. RAE-Revista de Administração de Empresas, 31(3), 73-82. Récupéré de http://bibliotecadigital.fgv.br/ojs/index. $\mathrm{php} / \mathrm{rae} /$ article/view/38605
Freitas, M. E. de. (1999a). Cultura organizacional: Formação, tipologias e impacto. São Paulo, SP: Editora Atlas.

Freitas, M. E. de. (1999b). Cultura organizacional: Identidade, sedução e carisma? Rio de Janeiro, RJ: FGV.

Freitas, M. E. de. (2001). Assédio moral e assédio sexual: faces do poder perverso nas organizações. RAE-Revista de Administração de Empresas, 41(2), 8-19. doi: 10.1590/So03475902001000200002

Freitas, M. E. de. (2008b). Entrevista com Jean-François Chanlat. GVexecutivo 7(11), 62-69. doi: 10.1266o/gvexec. v7n1.2008.34087

Freitas, M.-E. de. (2018). Gérer la diversité au Brésil. In J.F. Chanlat, \& Özbilgin, M, (Dir.), Management et diversité: Comparaisons internationales (pp.115-162). Ste-Foy, Québec, Canada: Les Presses de l'Université Laval; Paris, France: Hermann.

Freitas, M. E. de, \& Dantas, M. (2011). Diversidade sexual e trabalho. São Paulo, SP: Cengage.

Freitas, M. E. de, \& Dantas, M. (2014). L'étranger dans le groupe: Pont et porte de l'interculturel. In A.-M. Guénette, E. Mutabazi, S. Von O. Ottino, \& P. Pierre (Dir.), Management interculturel, altérité et identités (pp. 523-531). Paris, France: L'Harmattan.

Freitas, M. E. de, \& Motta, F. C. P. (2000). Vida psíquica e organização. Rio de Janeiro, RJ: Editora FGV.

Freyre, G. (1963). Casa grande e senzala. Brasília, DF: Ed. UnB.

Gantman, E,. Yousfi, H., \& Alcadipani, R. (2015). Challenging Anglo-Saxon dominance in management and organizational knowledge. RAE-Revista de Administração de Empresas, 55(2), 126-129. doi: 10.1590/S0034-759020150202V

Gauléjac, V. De. (2005). Gestão como doença social: Ideologia, poder gerencialista e fragmentação social. São Paulo, SP: Editora Ideias e Letras.

Gaviria, L. G. A., \& Molinier, P. (Éds.). (2011). El trabajo y la ética del cuidado. Bogotá, Colombia: La Carreta Social.

Girin, J. (2016). Langage, organisations, situations et agencements [avec la collaboration de Jean-François Chanlat, Hervé Dumez et Michèle Breton]. St-Foy, Québec, Canada: Presses de l'Université de Laval, Paris, France: Hermann.

Gonzáles-Miranda, D, R., \& Ramirez Martinez, G. (2018). Introducción: los estudiosorganizacionales en Latinoamérica. Una realidad fragmentada en busca de identidad Tratado de estudios organizacionales.(Volumen 1). Teorización sobre el campo, Medellin, Colombia: Editorial EAFIT, Mexico: Universidad Autónoma Metropolitana, Red Mexicana de Investigadores en Estudios Organizacionales, Londres: Sage, 21-40.

Gonzáles-Miranda, D, R., \& Ramirez Martinez, G. (2020). Tratado de estudios organizacionales. Volumen 2. Exploración de las temáticas, Medellin, Columbia: Editorial EAFIT, Mexico: Universidad Autónoma Metropolitana, Red Mexicana de Investigadores en Estudios Organizacionales, London, UK: Sage. 
Grange, J. (2000). Auguste Comte: La politique et la science. Paris, France: Éditions Odile Jacob.

Guedes, A., \& Faria, A. (Eds.). (2010). International management and international relations: A critical perspective from Latin America. New York, USA: Routledge.

Harzing, A-W. (2016, June). Do Google Scholar, Scopus and the Web of Science speak your language? EURAM Conference, Paris

Hatchuel, A. (2021, Janvier). La France, pionnière de la bonne gouvernance. Le Monde, 6 . Récupéré de https://www. lemonde.fr/emploi/article/2021/01/06/la-france-pionnierede-la-bonne-gouvernance_6065334_1698637.html

Ibarra-Colado, E. (2006). Organization studies and epistemic coloniality in Latin America: Thinking otherness from the margins. Organization, 13(4), 463-488. doi : $10.1177 / 1350508406065851$

Ibarra-Colado, E., Faria, A., \& Guedes, A. (2010). Introduction to the special issue on "Critical international management and international critical management: Perspectives from LatinAmerica". Critical Perspectives on International Business, 6(2/3), 86-96. doi: 10.1108/17422041011049923

Initiative d'Helsinki. (2019). Initiative d'Helsinki sur le multilinguisme dans la communication savante [Helsinki Initiative on Multilingualism in Scholarly Communication]. Récupéré de https://www.helsinki-initiative.org/fr

Instituto Brasileiro de Geografia e Estatistica. (2019). Sintese de Indicadores Sociais: uma análise das condições de vida da população brasileira. Rio de Janeiro, RJ: IBGE.

Jackson, T, \& Primecz, H. (2019). Cross-cultural management studies and the Englishization of scholarly communication: A paradox. International Journal of Cross Cultural Management, 19(2), 115-119. https://doi. org/10.1177/1470595819865095

Joly, A, (2004). Fiefs et entreprises en Amérique latine. Ste-Foy, Québec, Canada: Les Presses de l'université Laval.

Kirschner, A.-M., \& Gomes, E. R. (2014a). La sociologie brésilienne et l'entreprise à la fin du XXe siècle. Sociologies Pratiques, 3(3), 41-48. doi : 10.3917/sopr.hs01.0041

Kirschner, A.-M., \& Gomes, E. R. (2014b). Les sciences sociales brésiliennes aujourd'hui: Des questions structurelles aux nouvelles questions sociales. Sociologies Pratiques, 2(2), 123-134. doi: 10.3917/sopr.017.0123

Kury, L. (2003). Nation, races et fétichisme: La religion de l'humanité au Brésil. Revue d'Histoire des Sciences Humaines, 1(1), 125-137. doi: 10.3917/rhsh.008.0125

Lapassade, G. (1989). Grupos, organizações e instituições. Rio de Janeiro, RJ: Francisco Alves

Lapierre,L.(1989). Imaginário, Administração e Liderança. RAE Revista de Administração de Empresas, 29(4), 05-16. doi : 10.1590/So034-75901989000400002

Lapierre, L. (2005). Gérir e Criar. RAE-Revista de Administração de Empresas, 45(4), 108-117. doi : 10.1590/So03475902005000400008
Laufer, R. (1977). Crise de légitimité dans les grandes organisations. Revue Française de Gestion, 9 (1), 112-123.

Laufer, R., \& Paradeise, C. (1982). Le prince bureaucrate: Machiavel au pays du marketing. Paris, France: Flamarion.

Laville, J-L. (dir). (2011). Economie solidaire. Paris, France: Editions CNRS.

Lévesque, B., Bourque, G., \& Forgues, E. (1997, juil/décembre). La sociologie économique de langue française: Originalité et diversité des approches. Cahiers Internationaux de Sociologie, $103,265-294$.

Lima, M.-E. A. (1998). A psicopatologia do trabalho: Origens e desenvolvimentos recentes na França. Psicologia, Ciências e Profissão, 18(2), 10-15. doi: 10.1590/s141498931998000200003

Lima, M. E. A. (2013). Saúde mental e trabalho: Limites, desafios, obstáculos e perspectivas. Cadernos de Psicologia Social do Trabalho, 16(spe1), 91-98. doi: 10.11606/issn.1981-0490. v16ispe1p91-98

Lins, I. (1967). História do positivismo no Brasil. São Paulo, SP: Cia. Editora Nacional.

Lourau, R. (1975). A análise institucional. Petrópolis, RJ: Vozes.

Lussier, S., Chanlat, J-F. (2017). Les enseignants en gestion face aux nouvelles injonctions institutionnelles. Une étude FranceQuébec. Revue Française de Gestion, 43(267), 79-96. doi: $10.3166 / \mathrm{rfg} .2017 .00183$

Mandiola, M. (2010). Latin America's critical management? A liberation genealogy. Critical Perspectives on International Business, 6(2/3), 162-176. doi: 10.1108/17422041011049978

Martes, A.-C. B., Loureiro, M., Abramovay, S. M., \& Serafim, M. (2007). Apresentação Fórum - Sociologia econômica. RAEeletrônica, 6(1), Art 10. Récupéré de https://www.scielo.br/ $\mathrm{pdf} / \mathrm{raeel} / \mathrm{v6n} 1 /$ ao5v6n1.pdf

Martinet, A.-C. (Org.) (1990). Épistémologies et sciences de gestion. Paris, France: Economica.

Martinet, A.-C., \& Pesqueux, Y. (2013). Épistémologie des sciences de gestion. Paris, France: Vuibert.

Melo, M. C. De O. L. (1984). Processos de participação como meios não-instutionalizados dois postulados básicos de regulação de conflitos. RAE-Revista de Administração de Empresas, 24(4), 11-18. doi: 10.1590/So034-75901984000400004

Mello, M, S. B. de, Filho. (2019). Quarenta anos de Escola Francesa da Regulação: Entre o marxismo e o institucionalismo histórico. Economia e Sociedade, 28(1), 2529. doi: 10.1590/1982-3533.2019v28n1arto2

Merlo, A. C., \& Mendes, A. M. B. (2009). Perspectivas do uso da psicodinâmica do trabalho no Brasil: Teoria, pesquisa e ação. Cadernos de Psicologia Social do Trabalho, 12(2), 141-156. doi: 10.11606/issn.1981-0490.v12i2p141-156

Molinier, P. (2013). O trabalho e a psique: Uma introdução à psicodinâmica do trabalho (F. Soudant, Trad.). Brasília, DF: Paralelo 15. 
Motta, F. C. P. (1981). Burocracia e autogestão: A proposta de Proudhon. São Paulo, SP: Brasiliense.

Motta, F. C. P. (1983). A questão da formação do administrador. RAE-Revista de Administração de Empresas, 23(4), 53-55. doi: $10.1590 / \mathrm{s} 0034-75901983000400005$

Motta, F. C. P. (1984). Participação e co-gestão: Novas formas de administração. São Paulo, SP: Brasiliense.

Motta, F. C. P. (1988). As formas organizacionais do estado. RAERevista de Administração de Empresas, 28(4), 15-31. doi: 10.1590/So034-75901988000400003

Motta, F. C. P. (1990). Organização e poder. São Paulo, SP: Atlas.

Motta, F. C. P. (2002). Teoria geral da administração: Uma introdução. São Paulo, SP: Thompson Learning.

Motta, F. C. P. (2003). Teoria das organizações: Evolução e crítica. São Paulo, SP: Thompson Learning.

Motta, F. C. P., Alcadipani, R., \& Bresler, R. (2001). A valorização do estrangeiro como segregação nas organizações. Revista de Administração Contemporânea, 5(Edição Especial), 59-79. doi: $10.1590 /$ s1415-65552001000500004

Motta, F. C. P., \& Caldas, M. (1997). Cultura organizacional e cultura brasileira. São Paulo, SP: Atlas.

Nugent, P. (1993). Compte rendu de L'individu dans l'organisation: Les dimensions oubliées sous la direction de Jean-François Chanlat, Québec, Canada, Les Presses de l'Université Laval et les Éditions Eska, 1990, 842 p, Revue québécoise de science politique, (24), 186-191.

Nygaard, L. P. (2019). The institutional context of "linguistic injustice": Norwegian social scientists and situated multilingualis. Publications, 7(10), 1-13. Doi: 10.3390/ publications7010010

Orléan, A. (2004). Analyse économique des conventions. Paris, France: Quadrige, PUF.

Padioleau, J.-G. (1992, Juillet-septembre). Jean-François Clianlat, L'individu dans l'organisation, les dimensions oubliées, 1990. Sociologie du travail, 340 année, (3), pp. 384-385.

Pagès, M., Bonetti, M., \& Gauléjac, V. de. (1987). O poder das organizações. São Paulo, SP: Casa Editorial Atlas.

Paula, A. P. P. de. (2007). Guerreiro Ramos: Resgatando o pensamento de um sociólogo crítico das organizações. Organização \& Sociedade, 14(40), 169-188. doi: 10.1590/ S1984-92302007000100010

Paula, A. P. P. de, Maranhão, C. de A., Barreto, R. de O., \& Klechen, C. F. (2010). A tradição e a autonomia dos estudos organizacionais críticos no Brasil. RAE-Revista de Administração de Empresas, 50(1), 10-23. doi: 10.1590/ So034-75902010000100002

Perrow, C. (1976), Análise organizacional: Um enfoque sociológico. São Paulo, SP: Atlas.

Pesqueux, Y. (2008).Filosofia e Organizações - Coleção Debates em Administração. Rio de Janeiro, RJ: Cengage.
Pinot de Villechenon, F. (2003). La latinidad: una cuestión de afinidad cultural en las relaciones euro-latinoamericanas. In Cavallero, P, A., Buzón, R, P., Frenkel, D., \& Nocito, A. Koronis. Homenaje a Carlos A. Ronchi March. Buenos Aires, Argentina: Universidad de Buenos Aires, p. 395-409

Pinot de Villechenon, F. P., Chanlat, J.-F., \& Rizzo, H. L. (2021). Dans quelle mesure la perception de proximité culturelle s'ajustet-elle au contact avec le marché cible? Les enseignements d'une recherche menée auprès de dirigeants de PME sudeuropéennes au Brésil. Management International, 25(6) (sous presse).

Rambaud, A., Richard, J. (2021). Philosophie d'une écologie anticapitaliste: Pour un nouveau modèle de gestion écologique. Ste-Foy, Québec, Canada: Les Presses de l'Université Laval, Paris, France: Hermann.

Ramirez Martinez, G., \& Gonzáles-Miranda, D, R. (2018). Tratado de estudios organizacionales. Volumen 1. Teorización sobre el campo, Medellin, Columbia. Editorial EAFIT, Universidad Autónoma Metropolitana, Red Mexicana de Investigadores en Estudios Organizacionales, Sage

Ramos, A. G. (1981/1989). A nova ciência das organizações: Uma reconceituação da Riqueza das Nações ( $2^{\mathrm{a}}$ ed.). Rio de Janeiro, RJ: Editora da FGV.

Ramos, G. (1983). Administração e contexto brasileiro: Esboço de uma teoria geral da administração ( $2^{\mathrm{a}}$ ed.). Rio de Janeiro, RJ: Editora da Fundação Getulio Vargas.

Ramos, G. (1984). Modelos de Homen e teoria administrativa. Revista de Administração Pública, 18(2), 3-12. Récupéré de http://bibliotecadigital.fgv.br/ojs/index.php/rap/article/ view/10559

Reynaud, J.-D. (1989). Les règles du jeu: l'Action collective et la régulation sociale. Paris, France: Armand Colin.

Richebé, N., Favereau, O., Livian, Y., Bréchet, J.-P., Taskin, L., Havard, C., \& Croset, N. (2020). La théorie de la régulation sociale: Hommage à Jean-Daniel Reynaud. Revue française de gestion, 6(6), 105-128. doi: 10.3166/rfg.2020.00437

Rossi, A., \& Passos, E. (2014). Análise institucional: Revisão conceitual e nuances da pesquisa-intervenção no Brasil. Revista EPOS, 5(1), 156-181.

Rouanet, S. P. (2010). Quelques remarques sur l'influence de la philosophie française au Brésil. Synergies, Brésil numéro spécial (2), 41-46. Récupéré de https://gerflint.fr/Base/ Bresil_special2/Rouanet.pdf

Rouquié A. (1987). Amérique latine: introduction à l'Extrême Occident. Paris, France: Seuil,

Sainsaulieu, R. (1977/2014). L'Identité au travail. Paris, France: Presses de la Fondation des Sciences Politiques.

Sainsaulieu, R. (Dir.). (1990). L'entreprise: Une affaire de société. Paris, France: Presses de la Fondation des Sciences Politiques.

Sainsaulieu, R. (1997). Sociologie de l'entreprise. Paris, France: Presses de la Fondation des Sciences Politiques.

Sainsaulieu, R. (2002). Des sociétés en mouvement. Paris, France: Desclée de Brouwer. 
Sainsaulieu, R., \& Kirschner, A-M. (2006). Sociologia da Empresa Organização Poder, Cultura e Desenvolvimento no Brasil. Rio, Brasil: DP \& A Editora.

Sainsaulieu, R.,\& Segrestin, D. (1986, Juillet-septembre). Vers une théorie sociologique de l'entreprise. In: Sociologie du travail, 280 année, (3), pp. 335-352, Retour sur l'entreprise.

Salais, R., Thévenot, L.(éd.).(1986). Le travail : marché, règles et conventions. Paris, France: Economica.

Saussois, J. M. (2016). Les Organisations: Etat des savoirs, Paris, Editions Sciences Humaines.

Salvador, P. (2011, janeiro). Entrevista com NorbertAlter. Pequenas Empresas \& Grandes Negócios, 264. Récupéré de http:// revistapegn.globo.com/Revista/Common/o,,EMl199915 17172,00-ENTREVISTA+COM+NORBERT+ALTER+INTEGRA.html

Sciences Humaines, (1998, Mars-Avril). Comprendre les organisations, 20. Récupéré de https://www.scienceshumaines. com/comprendre-les-organisations_fr_95.htm

Segnini, L., \& Alcadipani, R. (2014, maio/junho). Poder e resistências nas organizações: A propósito das contribuições de Fernando C. Prestes Motta. RAE-Revista de Administração de Empresas, 54(3), 341-347. doi: 10.1590/s0034$759020140309 \mathrm{~V}$

Segrestin, B.(1992). Sociologie de l'entreprise. Paris, France: Armand Colin.

Segrestin, B., \& Hatchuel, A. (2012). Refonder l'entreprise. Paris, France: Seuil.

Séguin, F.,\& Chanlat, J-F. (1983). L'analyse des organisations: une anthologie sociologique. Tome I. Montréal, Québec, Canada, Gaëtan Morin.

Serva, M. (1990). Contribuições para uma teoria organizacional brasileira. Revista de Administração Pública, 24(2), 10-21. Récupéré de http://bibliotecadigital.fgv.br/ojs/index.php/ $\mathrm{rap} /$ article/view/9047

Serva, M. (1992). A importação de metodologias administrativas no Brasil: uma análise semiológica. Revista de Administração Pública, 26(4), 128-144. Récupéré de http://bibliotecadigital. fgv.br/ojs/index.php/rap/article/view/8751

Serva, M. (1997a). A racionalidade substantiva demonstrada na prática administrativa. RAE-Revista de Administração de Empresas, 37(2), 18-30. doi: 10.1590/s003475901997000200003

Serva, M. (1997b). Abordagem substantiva e ação comunicativa: Uma complementaridade frutuosa para a teoria das organizações. Revista de Administração Pública, 31(2), 108134. Récupéré de http://bibliotecadigital.fgv.br/ojs/index. $\mathrm{php} / \mathrm{rap} /$ article/view/7940

Serva, M. (2002). Contribuições da sociologia econômica à teoria das organizações. Sociedade e Estado, 17(1), 106-122. doi: $10.1590 /$ s0102-69922002000100007

Serva, M. (2013). O surgimento e desenvolvimento da epistemologia da administração: Inferências sobre a contribuição ao aperfeiçoamento da teoria administrativa. RGO Revista Gestão Organizacional, 6(Edição especial), 51-64. doi: 10.22277/rgo.v6i3.1529
Serva, M. (2017a). Epistemología de la administración en Brasil: El estado del arte. Cadernos EBAPE.BR, 15(4), 741-750. doi: 10.1590/1679-395173209

Serva, M. (2017b). A nova ideologia econômica da ciência e a (re) politização do campo. Ciências em Debate, 2, 52-58.

Serva, M., \& Andion, M.-C. (2006). Teoria das organizações e a nova sociologia econômica: Um diálogo interdisciplinar. $R A E$ Revista de Administração de Empresas, 46(2), 10-20. doi: 10.1590/S0034-75902006000200002

Serva, M., \& Andion, M.-C. (2021). La contribution de l'oeuvre de Jean-François Chanlat au champ des études épistémologiques des sciences administratives brésiliennes. In G. Bouville, \& H. Yousfi, Les dimensions oubliées de la gestion. Anthropologie, management et organisation: Mélanges en l'honneur de JeanFrançois Chanlat. Ste-Foy, Québec, Canada, Les Presses de l'université Laval, Paris, France: Hermann (à paraître).

Serva, M., Caitano, D., Santos, L., \& Siqueira, G. (2015). A análise da racionalidade nas organizações: Um balanço do desenvolvimento de um campo de estudos no Brasil. Cadernos EBAPE. br, 13(3), 414-437. Récupéré de http://bibliotecadigital.fgv. br/ojs/index.php/cadernosebape/article/view/16344

Silva, J.-R. G. da. (2010). La dynamique identitaire entre organisations et individus. Revue Française de Gestion, 36(20), 185-204. doi: 10.3166/rfg.203.185-204

Siqueira, G., \& Serva, M. (2014, março). Tensão entre racionalidades na abordagem substantiva das organizações. IV Colóquio Internacional de Epistemologia e Sociologia da Ciência da Administração, Florianópolis, SC.

Sperb, M. P., \& Serva, M. (2018). Economia social e solidária, governança e turismo no âmbito do desenvolvimento territorial sustentável. Revista de Ciências da Administração, 20(50), 93-109. doi: 10.5007/2175-8077.2018v2on5op93

Stokes, P., Davoine., E \& Oiry, E. (Guest Editors), (2014). Special issue on organizations and organizing in a French context. International Journal of Organizational Analysis, 22(4), 1-3. doi: 10.1108/IJOA-08-2014-0794

Sznelwar, I. L. (2006). Alain Wisner: O desenvolvimento da ergonomia e do pensamento sobre o "trabalhar". Travailler, 1(15), 55-70. doi: 10.3917/trav.015.0055

Sznelwar, L. I., Uchida, S., \& Lancman, S. (2011). A subjetividade no trabalho em questão. Tempo Social, 23(1), 11-30. doi: 10.1590/S0103-20702011000100002

Terssac, G. de. (Ed.). (2003). La théorie de la régulation sociale de Jean-Daniel Reynaud. Paris, France: La Decouverte.

Thévenet, M. (1992). Auditoría de la cultura empresarial. Madrid, España: Ediciones Díaz de Santos.

Tietze, S., \& Dicks, P. (2012, May). The victorious English language: Hegemonic practices in the management academy. Journal of Management Inquiry, 22(1), 122-134. doi: 10.1177/1056492612444316

Tonelli, M. J. (2018). Revistas científicas em administração: 0 papel histórico da Revista de Administração de Empresas (RAE) na construção do campo acadêmico em administração no Brasil. Cadernos EBAPE.BR, 16(Edição Especial), 509-515. doi: 10.1590/1679-395173941 
Torres, O., \& Gonçalves, M. A. (1991). O indivíduo na organização: dimensões esquecidas. RAE-Revista de Administração de Empresas, 31(4), 107-108. doi: 10.1590/ So034-75901991000400011

Tourish, D., \& Willmott, H, C. (2015, February). In Defiance of Folly: Journal Rankings, mindless measures and the ABS Guide. Critical Perspectives of Accounting, 26, 37-46. doi: 10.1016/j.cpa.2014.02.004

Tragtenberg, M. (2005). Administração, poder e ideologia. São Paulo, SP: Unisep.

Tragtenberg, M. (2010). Burocracia e ideologia. São Paulo, SP: Unisep.

Tragtenberg, M. (2012). Teoria e ação libertárias. São Paulo, SP: Unisep.

Tsuda, Y. (2013). Speaking Against the Hegemony of English Problems, Ideologies and Solutions. In The Handbook of Critical Intercultural Communication, Nakayama, T., \& Halualani, R, T, London, UK: Basil Blackwell, 248-269.

Tyr, A. (2007). Le drapeau brésilien et Auguste Comte [Film documentaire, coproduction Évasion en vidéo, PDR, TVM Est Parisien].

Vasconcelos, I., \& Pinochet, L. (2004). Poder, tecnologia e controle burocrático: Uma análise crozieriana em uma empresa de informática do governo do estado do Paraná. Revista de Administração Pública, 38(3), 465-479. Récupéré de http:// bibliotecadigital.fgv.br/ojs/index.php/rap/article/view/6546

Vergara, S. C. (2006). Análise organizacional no Brasil: Em busca da independência intelectual. In J.-F. Chanlat, R. Fachin, \& T. Fischer, Análise organizacional: Perspectivas latinas (Vol. 1: Olhar histórico e constatações atuais, pp. 219-238). Porto Alegre, RS: Editora da UFRGS.

Vergara, S. C., \& Pinto, M. (2001). Referências teóricas em análise organizacional: Um estudo da nacionalidade dos artigos referenciados na literatura brasileira. Revista de Administração Contemporânea, 5(Edição Especial), 103-121. doi: 10.1590/S1415-65552001000500006

Waiandt, C. A. (2009). O ensino dos estudos organizacionais nos cursos de pós-graduação stricto sensu em administração (Tese de doutorado, Escola de Administração, Universidade Federal da Bahia, Salvador, BA).

Waiandt, C. A. (2018). Ensino-Aprendizagem na administração: Contribuições e desafios na trajetória de Tânia Fischer. Farol - Revista de Estudos Organizacionais e Sociedade, 5(12), 85125. doi: 10.25113/farol.v5i12.4797

Willmott, H. (2011). Journal List Fetishism and the Perversion of Scholarship: Reactivity and the ABS List. Organization, 18(4), 429-442.

\section{CONTRIBUTIONS DE L'AUTEUR}

L'auteur déclare sur l'honneur qu'il a réalisé lui-même ce travail; de la conceptualisation à l'approche théorique et méthodologique jusqu'à la rédaction finale. 